\title{
Controllability of the discrete-spectrum Schrödinger equation driven by an external field
}

\author{
Thomas Chambrion $^{\mathrm{a}, *}$, Paolo Mason ${ }^{\mathrm{a}, \mathrm{b}}$, Mario Sigalotti ${ }^{\mathrm{a}}$, Ugo Boscain ${ }^{\mathrm{c}}$ \\ a Institut Élie Cartan, UMR 7502 Nancy-Université/CNRS/INRIA, BP 239, 54506 Vandouvre-lès-Nancy, France \\ ${ }^{\mathrm{b}}$ IAC, CNR, Viale Del Policlinico, 13700161 Rome, Italy \\ ${ }^{\mathrm{c}}$ Le2i, CNRS, Université de Bourgogne, BP 47870, 21078 Dijon Cedex, France
}

Received 31 January 2008; received in revised form 6 May 2008; accepted 8 May 2008

Available online 27 June 2008

\begin{abstract}
We prove approximate controllability of the bilinear Schrödinger equation in the case in which the uncontrolled Hamiltonian has discrete non-resonant spectrum. The results that are obtained apply both to bounded or unbounded domains and to the case in which the control potential is bounded or unbounded. The method relies on finite-dimensional techniques applied to the Galerkin approximations and permits, in addition, to get some controllability properties for the density matrix. Two examples are presented: the harmonic oscillator and the 3D well of potential, both controlled by suitable potentials.

๔ 2008 L'Association Publications de l'Institut Henri Poincaré. Published by Elsevier B.V. All rights reserved.
\end{abstract}

\section{Résumé}

Nous montrons la contrôlabilité approchée de l'équation de Schrödinger bilinéaire dans le cas où l'hamiltonien non contrôlé a un spectre discret et non-résonnant. Les résultats obtenus sont valables que le domaine soit borné ou non, et que le potentiel de contrôle soit borné ou non. La preuve repose sur des méthodes de dimension finie appliquées aux approximations de Galerkyn du système. Ces méthodes permettent en plus d'obtenir des résultats de contrôlabilité des matrices de densité. Deux exemples sont présentés, l'oscillateur harmonique et le puits de potentiel en dimension trois, munis de potentiels de contrôle adéquats.

๔ 2008 L'Association Publications de l'Institut Henri Poincaré. Published by Elsevier B.V. All rights reserved.

\section{Keywords: Quantum control; Control of PDE; Approximate controllability; Bilinear Schrödinger equation; Galerkin approximation; Density} matrix

\section{Introduction}

In this paper we study the controllability of the bilinear Schrödinger equation. Its importance is due to applications to modern technologies such as Nuclear Magnetic Resonance, laser spectroscopy, and quantum information science (see for instance $[22,29,31,38]$ ).

\footnotetext{
The last author was partially supported by a FABER grant of Conseil régional de Bourgogne.

* Corresponding author.

E-mail addresses: Thomas.Chambrion@iecn.u-nancy.fr (T. Chambrion), p.mason@iac.cnr.it (P. Mason), mario.sigalotti@inria.fr (M. Sigalotti), ugo.boscain@u-bourgogne.fr (U. Boscain).
} 
Many controllability results are available when the state space is finite dimensional, e.g., for spin systems or for molecular dynamics when one neglects interactions with highly excited levels (see for instance $[8,19]$ ). When the state space is infinite-dimensional the controllability problem appears to be much more intricate. Some results are available when the control is the value of the wave function on some portion of the boundary or in some internal region of the domain (see [41] and references therein and the recent paper [39]).

However, from the point of view of applications the case in which the control appears in the Hamiltonian as an external field is much more interesting, since the wave function is not directly accessible in experiments and because of the postulate of collapse of the wave function. For instance, in nuclear magnetic resonance the control is a magnetic field, in laser spectroscopy and in many applications of photochemistry the control is a laser or a source of light.

In this paper we consider the controllability problem for the following bilinear system representing the Schrödinger equation driven by one external field

$$
i \frac{d \psi}{d t}(t)=\left(H_{0}+u(t) H_{1}\right) \psi(t) .
$$

Here the wave function $\psi$ evolves in an infinite-dimensional Hilbert space, $H_{0}$ is a self-adjoint operator called drift Hamiltonian (i.e. the Hamiltonian responsible for the evolution when the external field is not active), $u(t)$ is a scalar control function, and $H_{1}$ is a self-adjoint operator describing the interrelation between the system and the external field.

The reference case is the one in which the Hilbert space is $L^{2}(\Omega)$ where $\Omega$ is either $\mathbf{R}^{d}$ or a bounded domain of $\mathbf{R}^{d}$, and Eq. (1.1) reads

$$
i \frac{\partial \psi}{\partial t}(t, x)=(-\Delta+V(x)+u(t) W(x)) \psi(t, x),
$$

where $\Delta$ is the Laplacian (with Dirichlet boundary condition in the case in which $\Omega$ is bounded) and $V$ and $W$ are suitably regular functions defined on $\Omega$. However the setting of the paper covers more general cases (for instance $\Omega$ can be a Riemannian manifold and $\Delta$ the corresponding Laplace-Beltrami operator). Let us stress that the proposed approach allows to handle both cases where the control potential (i.e. $H_{1}$ in (1.1) or $W$ in (1.2)) is bounded or unbounded. Notice that in many situations the control potential happens to be unbounded. For instance if $\Omega=\mathbf{R}^{d}$ and the controlled external force depends on time, but is constant in space, then $W$ is linear and hence unbounded.

Besides the fact that one cannot expect exact controllability on the whole Hilbert sphere (see [10,40]) and some negative result (in particular [28,36]) only few approximate controllability results are available and concern mainly special situations. It should be mentioned, however, that several results on efficient steering of the Schrödinger equation without any controllability assumptions are available, e.g. [11,14,23]. (For optimal control results for finite dimensional quantum systems see, for instance, [15-17,26].)

In $[12,13]$ Beauchard and Coron study the controllability of a quantum particle in a $1 \mathrm{D}$ potential well with $W(x)=x$. Their results are highly non-trivial and are based on Coron's return method (see [18]) and Nash-Moser's theorem. In particular, they prove that the system is exactly controllable in the unit sphere of the Sobolev space $H^{7}$ (implying in particular approximate controllability in $L^{2}$ ). One of the most interesting corollaries of this result is exact controllability between eigenstates.

A different result is given in [1], where adiabatic methods are used to prove approximate controllability for systems having conical eigenvalue crossings in the space of controls.

Another controllability result has been proved by Mirrahimi in [27] using Strichartz estimates and concerns approximate controllability for a certain class of systems such that $\Omega=\mathbf{R}^{d}$ and whose drift Hamiltonian has mixed spectrum (discrete and continuous).

The aim of the present paper is to prove a general approximate controllability result for a large class of systems for which the drift Hamiltonian $H_{0}$ has discrete spectrum. Our main assumptions are that the spectrum of $H_{0}$ satisfies a non-resonance condition and that $H_{1}$ couples each pair of distinct eigenstates of $H_{0}$. Such assumptions happen to be generic in a suitable sense, as it will be discussed in a forthcoming paper.

We then apply the approximate controllability result to two classical examples, namely the harmonic oscillator and the $3 \mathrm{D}$ potential well, for suitable controlled potentials.

Our method is new in the framework of quantum control and relies on finite-dimensional techniques applied to the Galerkin approximations. A difficult point is to deduce properties of the original infinite-dimensional system from 
its finite-dimensional approximations. For the Navier-Stokes equations this program was successfully conducted by Agrachev and Sarychev in the seminal paper [5] (see also [3,35]).

A key ingredient of the proof is a time reparameterization that inverts the roles of $H_{0}$ and $H_{1}$ as drift and control operator. This operation is crucial since it permits to exploit for the Galerkin approximation the techniques developed in [2] for finite-dimensional systems on compact semisimple Lie groups. The passage from the controllability properties of the Galerkin approximations to those of the infinite-dimensional system heavily relies on the fact that the dynamics preserve the Hilbert sphere.

A feature of our method is that the infinite-dimensional system inherits, in a suitable sense, controllability results for the group of unitary transformations from those of the Galerkin approximations. This permits to extract controllability properties for the density matrix. Let us stress that, as it happens in finite dimension, controllability properties for the density matrix cannot in general be deduced from those of the wave function (see for instance [7]).

The paper is organized as follows. In Section 2 we present the general functional analysis setting and we state our main result (Theorem 2.4) for the control system (1.1). In Section 3 we show how this result applies to the Schrödinger equation (1.2) when $\Omega$ is both bounded or unbounded. Section 4 contains the proof of Theorem 2.4 and an estimate of the minimum time for approximately steering the system between two given states, that holds even if the system itself is not approximately controllable. In Section 5 we extend Theorem 2.4 to the controlled evolution of the density matrix (Theorem 5.2). Finally in Section 6 we show how Theorems 2.4 and 5.2 can be applied to specific cases. In particular, we show how to get controllability results even in cases in which $V$ does not satisfy the required non-resonance hypothesis, using perturbation arguments.

\section{Mathematical framework and statement of the main result}

Hereafter $\mathbf{N}$ denotes the set of strictly positive integers. Definition 2.1 below provides the abstract mathematical framework that will be used to formulate and prove the controllability results later applied to the Schrödinger equation (1.2). The hypotheses under which (1.2) fits the abstract framework are discussed in Section 3.

Definition 2.1. Let $\mathscr{H}$ be a complex Hilbert space and $U$ be a subset of $\mathbf{R}$. Let $A, B$ be two, possibly unbounded, operators on $\mathscr{H}$ with values in $\mathscr{H}$ and denote by $D(A)$ and $D(B)$ their domains. The control system $(A, B, U)$ is the formal controlled equation

$$
\frac{d \psi}{d t}(t)=A \psi(t)+u(t) B \psi(t), \quad u(t) \in U
$$

We say that $(A, B, U)$ is a skew-adjoint discrete-spectrum control system if the following conditions are satisfied: (H1) $A$ and $B$ are skew-adjoint, (H2) there exists an orthonormal basis $\left(\phi_{n}\right)_{n \in \mathbf{N}}$ of $\mathscr{H}$ made of eigenvectors of $A$, (H3) $\phi_{n} \in D(B)$ for every $n \in \mathbf{N}$.

In order to give a meaning to the evolution equation (2.1), at least when $u$ is constant, we should ensure that the sum $A+u B$ is well defined. The standard notion of sum of operators seen as quadratic forms (see [20]) is not always applicable under the sole hypotheses (H1)-(H3). An adapted definition of $A+u B$ can nevertheless be given as follows: hypothesis (H3) guarantees that the sum $A+u B$ is well defined on $V=\operatorname{span}\left\{\phi_{n} \mid n \in \mathbf{N}\right\}$. Any skew-Hermitian operator $C: V \rightarrow \mathscr{H}$ admits a unique skew-adjoint extension $\mathcal{E}(C)$. We identify $A+u B$ with $\mathcal{E}\left(\left.A\right|_{V}+\left.u B\right|_{V}\right)$.

Let us notice that when $A+u B$ is well defined as sum of quadratic forms and is skew-adjoint then the two definitions of sum coincide. This happens in particular for the Schrödinger equation (1.2) in most physically significant situations (see Section 3).

A crucial consequence of what precedes is that for every $u \in U$ the skew-adjoint operator $A+u B$ generates a group of unitary transformations $e^{t(A+u B)}: \mathscr{H} \rightarrow \mathscr{H}$. In particular, the unit sphere $\mathcal{S}$ of $\mathscr{H}$ satisfies $e^{t(A+u B)}(\mathcal{S})=\mathcal{S}$ for every $u \in U$ and every $t \geqslant 0$.

Due to the dependence of the domain $D(A+u B)$ on $u$, the solutions of (2.1) cannot in general be defined in classical (strong, mild or weak) sense. Let us mention that, in some relevant cases in which the spectrum of $A$ has a non-trivial continuous component the solution can be defined as in $[30,34]$ by means of Strichartz estimates. 
We will say that the solution of (2.1) with initial condition $\psi_{0} \in \mathscr{H}$ and corresponding to the piecewise constant control $u:[0, T] \rightarrow U$ is the curve $t \mapsto \psi(t)$ defined by

$$
\psi(t)=e^{\left(t-\sum_{l=1}^{j-1} t_{l}\right)\left(A+u_{j} B\right)} e^{t_{j-1}\left(A+u_{j-1} B\right)} \cdots e^{t_{1}\left(A+u_{1} B\right)}\left(\psi_{0}\right),
$$

where $\sum_{l=1}^{j-1} t_{l} \leqslant t<\sum_{l=1}^{j} t_{l}$ and $u(\tau)=u_{j}$ if $\sum_{l=1}^{j-1} t_{l} \leqslant \tau<\sum_{l=1}^{j} t_{l}$. Notice that such a $\psi(\cdot)$ satisfies, for every $n \in \mathbf{N}$ and almost every $t \in[0, T]$, the differential equation

$$
\frac{d}{d t}\left\langle\psi(t), \phi_{n}\right\rangle=-\left\langle\psi(t),(A+u(t) B) \phi_{n}\right\rangle
$$

Remark 2.2. The notion of solution introduced above makes sense in very degenerate situations and can be enhanced when $B$ is bounded. Indeed, well-known results assert that in this case if $u \in L^{1}([0, T], U)$ then there exists a unique weak (and mild) solution $\psi \in \mathcal{C}([0, T], \mathscr{H})$ which coincides with the curve (2.2) when $u$ is piecewise constant. Moreover, if $\psi_{0} \in D(A)$ and $u \in \mathcal{C}^{1}([0, T], U)$ then $\psi$ is differentiable and it is a strong solution of (2.1). (See [10] and references therein.)

Definition 2.3. Let $(A, B, U)$ be a skew-adjoint discrete-spectrum control system. We say that $(A, B, U)$ is approximately controllable if for every $\psi_{0}, \psi_{1} \in \mathcal{S}$ and every $\varepsilon>0$ there exist $k \in \mathbf{N}, t_{1}, \ldots, t_{k}>0$ and $u_{1}, \ldots, u_{k} \in U$ such that

$$
\left\|\psi_{1}-e^{t_{k}\left(A+u_{k} B\right)} \cdots e^{t_{1}\left(A+u_{1} B\right)}\left(\psi_{0}\right)\right\|<\varepsilon .
$$

Let, for every $n \in \mathbf{N}, i \lambda_{n}$ denote the eigenvalue of $A$ corresponding to $\phi_{n}\left(\lambda_{n} \in \mathbf{R}\right)$. The main result of the paper is

Theorem 2.4. Let $\delta>0$ and $(A, B,(0, \delta))$ be a skew-adjoint discrete-spectrum control system. If the elements of the sequence $\left(\lambda_{n+1}-\lambda_{n}\right)_{n \in \mathbf{N}}$ are $\mathbf{Q}$-linearly independent and if $\left\langle B \phi_{n}, \phi_{n+1}\right\rangle \neq 0$ for every $n \in \mathbf{N}$, then $(A, B,(0, \delta))$ is approximately controllable.

Recall that the elements of the sequence $\left(\lambda_{n+1}-\lambda_{n}\right)_{n \in \mathbf{N}}$ are said to be Q-linearly independent if for every $N \in \mathbf{N}$ and $\left(q_{1}, \ldots, q_{N}\right) \in \mathbf{Q}^{N} \backslash\{0\}$ one has $\sum_{n=1}^{N} q_{n}\left(\lambda_{n+1}-\lambda_{n}\right) \neq 0$.

The condition $\left\langle B \phi_{n}, \phi_{n+1}\right\rangle \neq 0$, preferred here for the easiness of its expression, can be replaced by a weaker one (namely, (4.4)), as detailed in Remark 4.2.

\section{Discrete-spectrum Schrödinger operators}

The aim of this section is to recall some classical results on Schrödinger operators. In particular we list here, among the numerous situations studied in the literature, some well-known sufficient conditions guaranteeing that the controlled Schrödinger equation (1.2) satisfies the assumptions of Definition 2.1.

Theorem 3.1. (See [21, Theorem 1.2.2].) Let $\Omega$ be an open and bounded subset of $\mathbf{R}^{d}$ and $V \in L^{\infty}(\Omega, \mathbf{R})$. Then $-\Delta+V$, with Dirichlet boundary conditions, is a self-adjoint operator with compact resolvent. In particular $-\Delta+V$ has discrete spectrum and admits a family of eigenfunctions in $H^{2}(\Omega, \mathbf{R}) \cap H_{0}^{1}(\Omega, \mathbf{R})$ which forms an orthonormal basis of $L^{2}(\Omega, \mathbf{C})$.

Theorem 3.2. (See [32, Theorems XIII.69 and XIII.70].) Let $\Omega=\mathbf{R}^{d}$ and $V \in L_{\mathrm{loc}}^{1}\left(\mathbf{R}^{d}, \mathbf{R}\right)$ be bounded from below and such that

$$
\lim _{|x| \rightarrow \infty} V(x)=+\infty
$$

Then $-\Delta+V$, defined as a sum of quadratic forms, is a self-adjoint operator with compact resolvent. In particular $-\Delta+V$ has discrete spectrum and admits a family of eigenfunctions in $H^{2}\left(\mathbf{R}^{d}, \mathbf{R}\right)$ which forms an orthonormal basis of $L^{2}\left(\mathbf{R}^{d}, \mathbf{C}\right)$. Moreover, for every eigenfunction $\phi$ of $-\Delta+V$ and for every $a>0, x \mapsto e^{a\|x\|} \phi(x)$ belongs to $L^{2}\left(\mathbf{R}^{d}, \mathbf{C}\right)$. 
In the following, we call controlled Schrödinger equation the partial differential equation

$$
i \frac{\partial \psi}{\partial t}(t, x)=(-\Delta+V+u W) \psi(t, x)
$$

where $\psi: I \times \Omega \rightarrow \mathbf{C}, \Omega$ is an open subset of $\mathbf{R}^{d}, I$ is a subinterval of $\mathbf{R}$ and, in the case in which $\Omega$ is bounded, $\left.\psi\right|_{I \times \partial \Omega}=0$. The correct functional analysis framework for this equation is specified below.

The following corollary, which is a straightforward consequence of the results recalled above, states that the assumptions of Definition 2.1 are fulfilled by the operators appearing in the controlled Schrödinger equation under natural hypotheses.

Corollary 3.3. Let $\Omega$ be an open subset of $\mathbf{R}^{d}, V, W$ be two real-valued functions defined on $\Omega$, and $U$ be a subset of $\mathbf{R}$. Assume either that (i) $\Omega$ is bounded, $V, W$ belong to $L^{\infty}(\Omega, \mathbf{R})$ or that (ii) $\Omega=\mathbf{R}^{d}, V, W$ belong to $L_{\text {loc }}^{1}\left(\mathbf{R}^{d}, \mathbf{R}\right)$, the growth of $W$ at infinity is at most exponential and, for every $u \in U, \lim _{\|x\| \rightarrow+\infty}(V(x)+u W(x))=$ $+\infty$ and $\inf _{x \in \mathbf{R}^{d}}(V(x)+u W(x))>-\infty$. Let $\mathscr{H}$ be equal to $L^{2}(\Omega, \mathbf{C})$ and $D(A)$ be equal to $H^{2}(\Omega, \mathbf{C}) \cap H_{0}^{1}(\Omega, \mathbf{C})$ in case (i) and to $H^{2}(\Omega, \mathbf{C})$ in case (ii). Let, moreover, $A$ be the differential operator $-i(-\Delta+V)$ and $B$ be the multiplication operator $-i W$. Then $(A, B, U)$ is a skew-adjoint discrete-spectrum control system, called the controlled Schrödinger equation associated with $\Omega, V, W$ and $U$.

Since the controlled Schrödinger equation is a skew-adjoint discrete-spectrum control system, it makes sense to apply Theorem 2.4 to it. The result is the following theorem.

Theorem 3.4. Let $\Omega, V, W$ and $U$ satisfy one of the hypotheses (i) or (ii) of Corollary 3.3. Denote by $\left(\lambda_{k}\right)_{k \in \mathbf{N}}$ the sequence of eigenvalues of $-\Delta+V$ and by $\left(\phi_{k}\right)_{k \in \mathbf{N}}$ an orthonormal basis of $L^{2}(\Omega, \mathbf{C})$ of corresponding real-valued eigenfunctions. Assume, in addition to (i) or (ii), that $U$ contains the interval $(0, \delta)$ for some $\delta>0$, that the elements of $\left(\lambda_{k+1}-\lambda_{k}\right)_{k \in \mathbf{N}}$ are $\mathbf{Q}$-linearly independent, and that $\int_{\Omega} W(x) \phi_{k} \phi_{k+1} d x \neq 0$ for every $k \in \mathbf{N}$. Then the controlled Schrödinger equation associated with $\Omega, V, W$ and $U$ is approximately controllable.

As stressed just after the statement of Theorem 2.4, the condition $\int_{\Omega} W(x) \phi_{k} \phi_{k+1} d x \neq 0$ could be replaced by a weaker one (see Remark 4.2).

\section{Proof of Theorem 2.4}

The proof of Theorem 2.4 is split in several steps. First, in Section 4.1 the controllability problem is transformed, thanks to a time-reparameterization, into an equivalent one where $A$ and $B$ play the role of controlled dynamics and drift, respectively. Then, in Section 4.2, we prove a controllability result for the Galerkin approximations of this equivalent system. In Section 4.3 we show how to lift the controllability properties from a Galerkin approximation to an higher-dimensional one. Section 4.4 makes the link between finite-dimensional and infinite-dimensional controllability properties and completes the proof.

Finally, in Section 4.5, as a byproduct of the arguments of the proof, we get a lower bound on the minimum steering time.

\subsection{Time-reparameterization}

First remark that, if $u \neq 0, e^{t(A+u B)}=e^{t u((1 / u) A+B)}$. Theorem 2.4 is therefore equivalent to the following property: if the elements of the sequence $\left(\lambda_{n+1}-\lambda_{n}\right)_{n \in \mathbf{N}}$ are $\mathbf{Q}$-linearly independent and if $\left\langle B \phi_{n}, \phi_{n+1}\right\rangle \neq 0$ for every $n \in \mathbf{N}$, then for every $\delta, \varepsilon>0$ and every $\psi_{0}, \psi_{1} \in \mathcal{S}$ there exist $k \in \mathbf{N}, t_{1}, \ldots, t_{k}>0$ and $u_{1}, \ldots, u_{k}>\delta$ such that

$$
\left\|\psi_{1}-e^{t_{k}\left(u_{k} A+B\right)} \cdots e^{t_{1}\left(u_{1} A+B\right)}\left(\psi_{0}\right)\right\|<\varepsilon
$$

In other words, the system for which the roles of $A$ and $B$ as drift and controlled field are inverted, namely,

$$
\frac{d \psi}{d t}(t)=u(t) A \psi(t)+B \psi(t), \quad u(t) \in U,
$$

is approximately controllable provided that the control set $U$ contains a half-line. The notion of solution of (4.2) corresponding to a piecewise constant control function is defined as in (2.2). 


\subsection{Controllability of the Galerkin approximations}

Let, for every $j, k \in \mathbf{N}, b_{j k}=\left\langle B \phi_{j}, \phi_{k}\right\rangle$ and $a_{j k}=\left\langle A \phi_{j}, \phi_{k}\right\rangle=i \lambda_{j} \delta_{j k}$. (Recall that $\lambda_{j} \in \mathbf{R}$ and $\left\{i \lambda_{j} \mid j \in \mathbf{N}\right\}$ is the spectrum of $A$.) Define, for every $n \in \mathbf{N}$, the two complex-valued $n \times n$ matrices $A^{(n)}=\left(a_{j k}\right)_{1 \leqslant j, k \leqslant n}$ and $B^{(n)}=\left(b_{j k}\right)_{1 \leqslant j, k \leqslant n}$. The Galerkin approximation of (4.2) at order $n$ (with respect to the basis $\left.\left(\phi_{k}\right)_{k \in \mathbf{N}}\right)$ is the finitedimensional control system

$$
\frac{d x}{d t}=u A^{(n)} x+B^{(n)} x, \quad x \in \mathcal{S}_{n}, u>\delta,
$$

where $\mathcal{S}_{n}$ denotes the unit sphere of $\mathbf{C}^{n}$. Notice that the system is well defined since, by construction, $A^{(n)}$ and $B^{(n)}$ are skew-Hermitian matrices.

We say the $\left(\Sigma_{n}\right)$ is controllable if for every $x_{0}, x_{1} \in \mathcal{S}_{n}$ there exist $k \in \mathbf{N}, t_{1}, \ldots, t_{k}>0$ and $u_{1}, \ldots, u_{k}>\delta$ such that

$$
x_{1}=e^{t_{k}\left(u_{k} A^{(n)}+B^{(n)}\right)} \cdots e^{t_{1}\left(u_{1} A^{(n)}+B^{(n)}\right)} x_{0} .
$$

We recall that a $n \times n$ matrix $C=\left(c_{j k}\right)_{1 \leqslant j, k \leqslant n}$ is said to be connected if for every pair of indices $j, k \in\{1, \ldots, n\}$ there exists a finite sequence $r_{1}, \ldots, r_{l} \in\{1, \ldots, n\}$ such that $c_{j r_{1}} c_{r_{1} r_{2}} \cdots c_{r_{l-1} r_{l}} c_{r_{l} k} \neq 0$. (In the literature connected matrices are sometimes called einfach, or irreducible, or inseparable.) The following proposition is in the spirit of the controllability results obtained in [2] and [40].

Proposition 4.1. Let $\mathfrak{A}=\left(\alpha_{j k}\right)_{j, k=1}^{n}, \mathfrak{B}=\left(\beta_{j k}\right)_{j, k=1}^{n}$ be two skew-symmetric $n \times n$ matrices and assume that $\mathfrak{A}$ is diagonal and $\mathfrak{B}$ is connected. Assume moreover that $\left|\alpha_{j j}-\alpha_{k k}\right| \neq\left|\alpha_{l l}-\alpha_{m m}\right|$ if $\{j, k\} \neq\{l, m\}$. Then the control system $(\bar{\Sigma}): \dot{x}=u \mathfrak{A} x+\mathfrak{B} x$ is controllable in $\mathcal{S}_{n}$ with piecewise constant controls $u: \mathbf{R} \rightarrow \bar{U}$, provided that $\bar{U}$ contains at least two points.

Proof. For every $1 \leqslant j, k \leqslant n$ let $e_{j k}$ be the $n \times n$ matrix whose entries are all equal to zero except the one at line $j$ and column $k$ which is equal to 1 .

Define for every $p \in \mathbf{N}$ the iterated matrices commutator $M_{p}=\operatorname{ad}_{\mathfrak{A}}^{p}(\mathfrak{B})$. (Recall the usual notation $\operatorname{ad}_{X}(Y)=$ $[X, Y]=X Y-Y X$ for the adjoint operator associated with $X$.) A simple induction on $p$ shows that the matrix $M_{p}$ has the expression

$$
M_{p}=\sum_{l, m=1}^{n}\left(\alpha_{l l}-\alpha_{m m}\right)^{p} \beta_{l m} e_{l m} .
$$

Fix two indices $j \neq k$ such that $1 \leqslant j, k \leqslant n$ and $\beta_{j k} \neq 0$. Since, by hypothesis, $\left(\alpha_{j j}-\alpha_{k k}\right)^{2} \neq\left(\alpha_{l l}-\alpha_{m m}\right)^{2}$ as soon as $\{j, k\} \neq\{l, m\}$, there exists some polynomial $P_{j k}$ with real coefficients such that $P_{j k}\left(\left(\alpha_{j j}-\alpha_{k k}\right)^{2}\right)=1$ and $P_{j k}\left(\left(\alpha_{l l}-\alpha_{m m}\right)^{2}\right)=0$ for all $\{l, m\} \neq\{j, k\}, 1 \leqslant l, m \leqslant n$.

Let us define $\left(c_{h}\right)_{h}$ as the coefficients of $P_{j k}$, i.e., $P_{j k}(X)=\sum_{h=0}^{d} c_{h} X^{h}$. Define moreover the matrix $N_{j k}=$ $\sum_{h=0}^{d} c_{h} M_{2 h}$. By construction $N_{j k}=\sum_{l, m=1}^{n} \beta_{l m} e_{l m} P_{j k}\left(\left(\alpha_{l l}-\alpha_{m m}\right)^{2}\right)=\beta_{j k} e_{j k}-\overline{\beta_{j k}} e_{k j}$. Therefore, the commutator $\left[\mathfrak{A}, N_{j k}\right]$ is equal to $\left(\alpha_{j j}-\alpha_{k k}\right)\left(\beta_{j k} e_{j k}+\overline{\beta_{j k}} e_{k j}\right)$ and so the Lie algebra generated by $\mathfrak{A}$ and $\mathfrak{B}$ contains the two elementary anti-Hermitian matrices $E_{j k}=e_{j k}-e_{k j}$ and $F_{j k}=i\left(e_{j k}+e_{k j}\right)$.

Notice now that, for every $1 \leqslant j, k, h, m \leqslant n, e_{j k} e_{h m}=\delta_{k h} e_{j m}$ and therefore

$$
\begin{aligned}
& {\left[E_{j k}, E_{k m}\right]=E_{j m}+\delta_{k m} E_{k j}+\delta_{k j} E_{m k},} \\
& {\left[E_{j k}, F_{j k}\right]=2 i\left(e_{j j}-e_{k k}\right) .}
\end{aligned}
$$

It follows from the definition of connected matrix and the relation $\left[\mathfrak{A}, E_{j k}\right]=i\left(\alpha_{k k}-\alpha_{j j}\right) F_{j k}$ that the Lie algebra generated by $\mathfrak{A}$ and $\mathfrak{B}$ contains the matrices $E_{j k}, F_{j k}$ and $i\left(e_{j j}-e_{k k}\right)$ for every $j \neq k$. Therefore

$$
\mathfrak{s u}(n) \subseteq \operatorname{Lie}(\mathfrak{A}, \mathfrak{B}) .
$$

Fix $\bar{x} \in \mathcal{S}_{n}$ and consider the submersion $\mathcal{P}: \mathrm{SU}(n) \rightarrow \mathcal{S}_{n}$ defined by $\mathcal{P}(g)=g \bar{x}$. Since

$$
T_{\mathcal{P}(g)}\left(\mathcal{S}_{n}\right)=\mathcal{P}_{*}\left(T_{g} \mathrm{SU}(n)\right)=\mathcal{P}_{*}(\mathfrak{s u}(n) g)=\mathfrak{s u}(n) g \bar{x}=\mathfrak{s u}(n) \mathcal{P}(g),
$$


then the evaluation at $x=\mathcal{P}(g)$ of the Lie algebra generated by $\mathfrak{A}$ and $\mathfrak{B}$ contains the whole space $T_{x} \mathcal{S}_{n}$. Since for any $u \in \bar{U}$ and any $t \in \mathbf{R}$ the flow $e^{t(u \mathfrak{A}+\mathfrak{B})}: \mathcal{S}_{n} \rightarrow \mathcal{S}_{n}$ is volume-preserving then $(\bar{\Sigma})$ is controllable (see [4, Cor. 8.6, Prop. 8.14, Th. 8.15]).

The condition $b_{j, j+1} \neq 0$ for every $j \in \mathbf{N}$ appearing in Theorem 2.4 clearly ensures that every matrix $B^{(n)}$ is connected. Proposition 4.1, applied to $A^{(n)}=\mathfrak{A}$ and $B^{(n)}=\mathfrak{B}$, implies therefore that $\left(\Sigma_{n}\right)$ is controllable.

Remark 4.2. In the following we can replace the assumption that $b_{j, j+1} \neq 0$ for every $j \in \mathbf{N}$ with the weaker one that $B^{(n)}$ is frequently connected, that is,

$\forall j \in \mathbf{N}, \quad \exists k \geqslant j \mid B^{(k)}$ is connected.

Notice, as a partial counterpart, that if there exists a non-empty and proper subset $\Xi$ of $\mathbf{N}$ such that for every $j \in \Xi$ and $k \in \mathbf{N} \backslash \Xi$ the coefficient $b_{j k}$ is equal to zero (i.e., the infinite-dimensional matrix $\left(b_{l m}\right)_{l, m \in \mathbf{N}}$ is non-connected) then the control system (2.1) is not approximately controllable. Indeed, the subspace $\operatorname{span}\left\{\phi_{k} \mid k \in \Xi\right\}$ is invariant for the dynamics of $A+u B$ for every $u \in U$ and has non-trivial (invariant) orthogonal.

\subsection{Approximate controllability in higher-dimensional projections}

Fix $\delta, \varepsilon>0$ and $\psi_{0}, \psi_{1} \in \mathcal{S}$. For every $n \in \mathbf{N}$, let $\Pi_{n}: \mathscr{H} \rightarrow \mathscr{H}$ be the orthogonal projection on the space $\operatorname{span}\left(\phi_{1}, \ldots, \phi_{n}\right)$ and $\bar{\Pi}_{n}: \mathscr{H} \rightarrow \mathbf{C}^{n}$ be the map that associates to an element of $\mathscr{H}$ the vector of its first $n$ coordinates with respect to the basis $\left(\phi_{m}\right)_{m \in \mathbf{N}}$. Choose $n$ such that

$$
\left\|\psi_{j}-\Pi_{n}\left(\psi_{j}\right)\right\|<\varepsilon \quad \text { for } j=0,1 \text {. }
$$

Thanks to (4.4) we can assume, without loss of generality, that $\left(\Sigma_{n}\right)$ is controllable. Let $u:[0, T] \rightarrow(\delta, \infty)$ be the piecewise constant control driving $\xi_{0} /\left\|\xi_{0}\right\|$ to $\xi_{1} /\left\|\xi_{1}\right\|$ where $\xi_{j}=\bar{\Pi}_{n}\left(\psi_{j}\right)$ for $j=0,1$.

Let $\mu>0$ be a constant which will be chosen later small enough, depending on $T, n$, and $\varepsilon$. Notice that for every $j \in \mathbf{N}$ the hypothesis that $\phi_{j}$ belongs to $D(B)$ implies that the sequence $\left(b_{j k}\right)_{k \in \mathbf{N}}$ is in $l^{2}$. It is therefore possible to choose $N \geqslant n$ such that

$$
\sum_{k>N}\left|b_{j k}\right|^{2}<\mu, \quad \text { for every } j=1, \ldots, n .
$$

If $t \mapsto X(t)$ is a solution of $\left(\Sigma_{N}\right)$ corresponding to a control function $U(\cdot)$, then $t \mapsto e^{-V(t) A^{(N)}} X(t)=Y(t)$, where $V(t)=\int_{0}^{t} U(\tau) d \tau$, is a solution of

$$
\dot{Y}(t)=e^{-V(t) A^{(N)}} B^{(N)} e^{V(t) A^{(N)}} Y(t) .
$$

Let us represent the matrix $e^{-v(t) A^{(N)}} B^{(N)} e^{v(t) A^{(N)}}$, where $v(t)=\int_{0}^{t} u(\tau) d \tau$, in block form as follows

$$
e^{-v(t) A^{(N)}} B^{(N)} e^{v(t) A^{(N)}}=\left(\begin{array}{cc}
M^{(n, n)}(t) & M^{(n, N-n)}(t) \\
M^{(N-n, n)}(t) & M^{(N-n, N-n)}(t)
\end{array}\right),
$$

where the superscripts indicate the dimensions of each block.

Claim 4.3. There exists a sequence of piecewise constant control functions $u_{k}:[0, T] \rightarrow(\delta, \infty)$ such that the sequence of matrix-valued curves

$$
t \mapsto M_{k}(t)=e^{-v_{k}(t) A^{(N)}} B^{(N)} e^{v_{k}(t) A^{(N)}},
$$

where $v_{k}(t)=\int_{0}^{t} u_{k}(\tau) d \tau$, converges to

$$
t \mapsto M(t)=\left(\begin{array}{cc}
M^{(n, n)}(t) & 0_{n \times(N-n)} \\
0_{(N-n) \times n} & M^{(N-n, N-n)}(t)
\end{array}\right)
$$

in the following integral sense

$$
\int_{0}^{t} M_{k}(\tau) d \tau \rightarrow \int_{0}^{t} M(\tau) d \tau
$$

as $k \rightarrow \infty$ uniformly with respect to $t \in[0, T]$. 
Proof. We will prove the claim taking $v(\cdot)$ piecewise constant, since every piecewise affine function can be approximated arbitrarily well in the $L^{\infty}$ topology by piecewise constant functions and because the map associating to $v(\cdot)$ the curve $t \mapsto \int_{0}^{t} M(\tau) d \tau$ is continuous with respect to the $L^{\infty}$ topology (taken both in its domain and its codomain).

Assume that $v(\cdot)$ is constantly equal to $w \in \mathbf{R}$ on $\left[t_{1}, t_{2}\right]$. Since $\lambda_{2}-\lambda_{1}, \ldots, \lambda_{N}-\lambda_{N-1}$ are $\mathbf{Q}$-linearly independent, then for every $s_{0} \in \mathbf{R}$ the curve

$$
\left(s_{0}, \infty\right) \ni s \mapsto\left(\left(\lambda_{1}-\lambda_{2}\right) s, \ldots,\left(\lambda_{1}-\lambda_{N}\right) s\right)
$$

projects onto a dense subset of the torus $\mathbf{R}^{N-1} / 2 \pi \mathbf{Z}^{N-1}$. Thus, there exist two sequences $w^{(m)} \nearrow+\infty$ and $z^{(m)} \nearrow+\infty$ such that

$$
\begin{array}{ll}
\left(\lambda_{1}-\lambda_{j}\right) w^{(m)}(\bmod 2 \pi) \longrightarrow\left(\lambda_{1}-\lambda_{j}\right) w(\bmod 2 \pi) & \text { for } 2 \leqslant j \leqslant N, \\
\left(\lambda_{1}-\lambda_{j}\right) z^{(m)}(\bmod 2 \pi) \longrightarrow\left(\lambda_{1}-\lambda_{j}\right) w(\bmod 2 \pi) & \text { for } 2 \leqslant j \leqslant n, \\
\left(\lambda_{1}-\lambda_{j}\right) z^{(m)}(\bmod 2 \pi) \longrightarrow\left(\lambda_{1}-\lambda_{j}\right) w+\pi(\bmod 2 \pi) & \text { for } n+1 \leqslant j \leqslant N,
\end{array}
$$

as $m$ tends to infinity. In particular the sequence of matrices $e^{-w^{(m)} A^{(N)}} B^{(N)} e^{w^{(m)}} A^{(N)}$ converges to $e^{-w A^{(N)}} B^{(N)} e^{w A^{(N)}}$

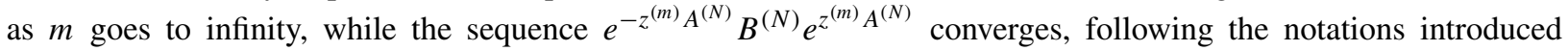
in (4.7), to

$$
\left(\begin{array}{cc}
M^{(n, n)} & -M^{(n, N-n)} \\
-M^{(N-n, n)} & M^{(N-n, N-n)}
\end{array}\right),
$$

where we dropped the dependence on $t$ of the different sub-matrices since $v$ is constant on $\left[t_{1}, t_{2}\right]$.

Fix $\bar{\delta}>\delta$. Consider a sequence $\left(\bar{v}_{k}\right)_{k \in \mathbf{N}}$ in $\mathbf{R}_{+}$(whose role will be clarified later) and define, for every $k \in \mathbf{N}$, a finite increasing sequence $\left(\theta_{l}^{k}\right)_{l=0, \ldots, k}$ with $\theta_{0}^{k}=\bar{v}_{k}, \theta_{l+1}^{k} \geqslant \theta_{l}^{k}+\bar{\delta}\left(t_{2}-t_{1}\right) / k^{2}$ for $0 \leqslant l<k$, and such that, for $l>0$, $\theta_{l}^{k}$ belongs to $\left(w^{(m)}\right)_{m \in \mathbf{N}}$ if $l$ is odd and to $\left(z^{(m)}\right)_{m \in \mathbf{N}}$ if $l$ is even. Define, moreover,

$$
\tau_{j}=t_{1}+\frac{j-1}{k}\left(t_{2}-t_{1}\right)
$$

for $j=1, \ldots, k+1$.

Consider the continuous function $v_{k}$ uniquely defined on $\left[t_{1}, t_{2}\right]$ by the conditions

$$
\begin{cases}v_{k}\left(t_{1}\right)=\bar{v}_{k}, & \\ v_{k}\left(\tau_{i}+\frac{t_{2}-t_{1}}{k^{2}}\right)=\theta_{i}^{k} & \text { for } i=1, \ldots, k, \\ \dot{v}_{k}(t)=\bar{\delta} & \text { if } t \in \bigcup_{i=1}^{k}\left(\tau_{i}+\frac{t_{2}-t_{1}}{k^{2}}, \tau_{i+1}\right), \\ \ddot{v}_{k}(t)=0 & \text { if } t \in \bigcup_{i=1}^{k}\left(\tau_{i}, \tau_{i}+\frac{t_{2}-t_{1}}{k^{2}}\right) .\end{cases}
$$

(See Fig. 1.) Define $\tilde{v}_{k}$ as the piecewise constant function that coincides with $\theta_{i}^{k}$ on $\left[\tau_{i}, \tau_{i+1}\right]$. On each interval $\left[\tau_{i}+\left(t_{2}-t_{1}\right) / k^{2}, \tau_{i+1}\right]$ the difference between $v_{k}$ and $\tilde{v}_{k}$ is bounded in absolute value by $\bar{\delta}\left(t_{2}-t_{1}\right) / k$. Therefore,

$$
\sup \left\{\left\|e^{-v_{k}(t) A^{(N)}} B^{(N)} e^{v_{k}(t) A^{(N)}}-e^{-\tilde{v}_{k}(t) A^{(N)}} B^{(N)} e^{\tilde{v}_{k}(t) A^{(N)}}\right\| \mid t \in \bigcup_{i=1}^{k}\left[\tau_{i}+\frac{t_{2}-t_{1}}{k^{2}}, \tau_{i+1}\right]\right\}
$$

goes to zero as $k$ goes to infinity.

Since $\left\|e^{-v A^{(N)}} B^{(N)} e^{\nu A^{(N)}}\right\|$ is uniformly bounded with respect to $v \in \mathbf{R}$ and the measure of $\bigcup_{i}\left[\tau_{i}, \tau_{i}+\frac{T}{k^{2}}\right]$ goes to 0 as $k$ goes to infinity, we have

$$
\int_{t_{1}}^{t}\left(e^{-v_{k}(\tau) A^{(N)}} B^{(N)} e^{v_{k}(\tau) A^{(N)}}-e^{-\tilde{v}_{k}(\tau) A^{(N)}} B^{(N)} e^{\tilde{v}_{k}(\tau) A^{(N)}}\right) d \tau \stackrel{k \rightarrow \infty}{\longrightarrow} 0 \quad \text { uniformly on }\left[t_{1}, t_{2}\right] .
$$

Moreover, by definition of the sequences $\theta_{i}^{k}, w^{(m)}$, and $z^{(m)}$, one has

$$
\int_{t_{1}}^{t} e^{-\tilde{v}_{k}(\tau) A^{(N)}} B^{(N)} e^{\tilde{v}_{k}(\tau) A^{(N)}} d \tau \stackrel{k \rightarrow \infty}{\longrightarrow} \int_{t_{1}}^{t} M(\tau) d \tau \quad \text { uniformly on }\left[t_{1}, t_{2}\right],
$$




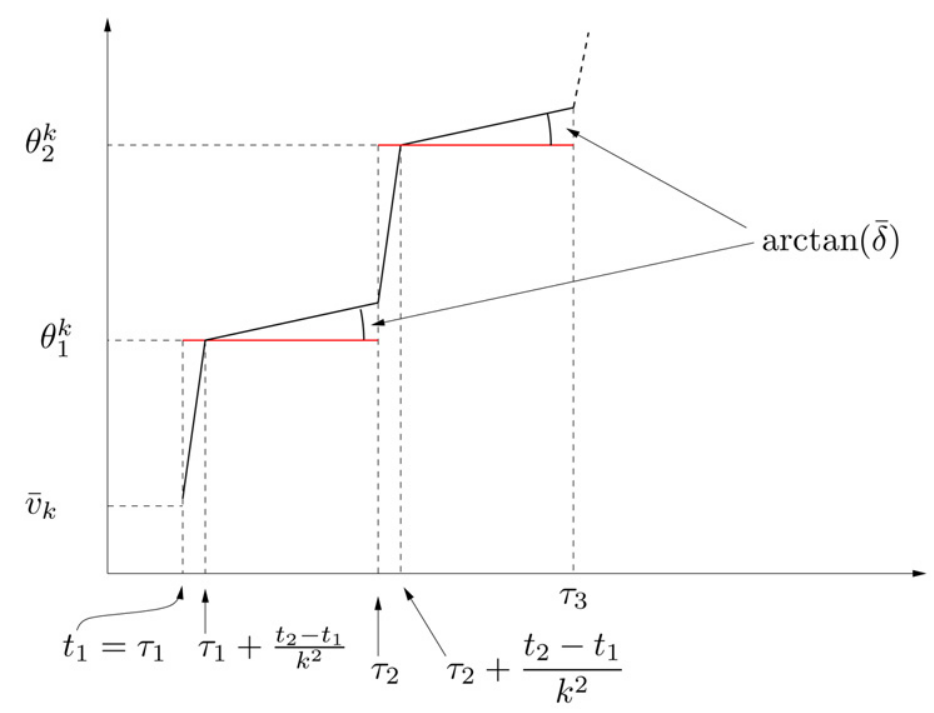

Fig. 1. The functions $v_{k}$ and $\tilde{v}_{k}$.

and therefore $e^{-v_{k}(t) A^{(N)}} B^{(N)} e^{v_{k}(t) A^{(N)}}$ converges in integral sense to $M(t)$ on $\left[t_{1}, t_{2}\right]$.

Finally, construct $u_{k}$ as follows: for $t_{1}=0$ define $u_{k}$ on $\left(t_{1}, t_{2}\right)$ as the derivative of $v_{k}$ (defined almost everywhere), where the $v_{k}$ 's correspond to the sequence of initial conditions $\bar{v}_{k}=0$ for every $k \in \mathbf{N}$. Then, on the second interval on which $v(\cdot)$ is constant, use as a new set of initial conditions for the approximation procedure the values $\bar{v}_{k}=v_{k}\left(t_{2}\right)$ and define again $u_{k}$ as the derivative of $v_{k}$. Iterating the procedure on the finite set of intervals covering $[0, T]$ on which $v(\cdot)$ is constant we obtain the required approximating sequence of piecewise constant control functions.

\subsection{Approximate controllability for the infinite-dimensional system}

Let $u_{k}$ and $M_{k}$ be defined as in Claim 4.3. The resolvent $R_{k}(t, s): \mathbf{C}^{N} \rightarrow \mathbf{C}^{N}$ of the linear time-varying equation

$$
\dot{Y}=M_{k}(t) Y
$$

converges, uniformly with respect to $(t, s)$, to the resolvent $R(t, s): \mathbf{C}^{N} \rightarrow \mathbf{C}^{N}$ of

$$
\dot{Y}=M(t) Y \text {. }
$$

(See, for instance, [4, Lemma 8.10].) Notice that $R(t, s)$ preserves the norms of both the vector formed by the first $n$ coordinates and the one formed by the last $N-n$.

Let, for every $k \in \mathbf{N}, \psi^{k}$ be the solution of (4.2) corresponding to $u_{k}$. We have the following approximation property.

Claim 4.4. For $k$ large enough,

$$
\left\|\Pi_{n}\left(e^{-v_{k}(T) A} \psi^{k}(T)\right)-\Pi_{n}\left(e^{-v(T) A} \psi_{1}\right)\right\|<2 \varepsilon,
$$

where $\varepsilon$ is the positive constant which has been fixed at the beginning of Section 4.3.

Proof. Define $q^{k}(t)=e^{-i v_{k}(t) A} \psi^{k}(t)$. According to (2.3) (more precisely, its counterpart for Eq. (4.2)), the components $q_{j}^{k}(t)=e^{-i \lambda_{j} v_{k}(t)}\left\langle\psi^{k}(t), \phi_{j}\right\rangle$ of $q^{k}(t)$ with respect to the basis of eigenvectors of $A$ satisfy for almost every $t \in[0, T]$

$$
\dot{q}_{j}^{k}(t)=\sum_{l=1}^{\infty} b_{j l} e^{i\left(\lambda_{l}-\lambda_{j}\right) v_{k}(t)} q_{l}^{k}(t) .
$$


Therefore, the curves $P^{k}(t)=\left(q_{1}^{k}(t), \ldots, q_{n}^{k}(t)\right)^{T}$ and $Q^{k}(t)=\left(q_{n+1}^{k}(t), \ldots, q_{N}^{k}(t)\right)^{T}$ satisfy

$$
\left(\begin{array}{c}
\dot{P}^{k}(t) \\
\dot{Q}^{k}(t)
\end{array}\right)=M_{k}(t)\left(\begin{array}{c}
P^{k}(t) \\
Q^{k}(t)
\end{array}\right)+\left(\begin{array}{c}
H^{k}(t) \\
I^{k}(t)
\end{array}\right)
$$

with $\left\|H^{k}\right\|_{\infty}<\sqrt{n \mu}$ (see (4.6)) and $\left\|I^{k}\right\|_{\infty}<C$ for $C=C(N)$ large enough.

Hence

$$
\left(\begin{array}{c}
P^{k}(t) \\
Q^{k}(t)
\end{array}\right)=R_{k}(t, 0) \bar{\Pi}_{N}\left(\psi_{0}\right)+\int_{0}^{t} R_{k}(s, t)\left(\begin{array}{c}
H^{k}(s) \\
I^{k}(s)
\end{array}\right) d s .
$$

Denote by $\bar{\Pi}_{n}^{N}$ the projection of $\mathbf{C}^{N}$ on its first $n$ coordinates and let

$$
L^{k}(t)=\bar{\Pi}_{n}^{N}\left(\int_{0}^{t} R_{k}(s, t)\left(\begin{array}{c}
H^{k}(s) \\
I^{k}(s)
\end{array}\right) d s\right) .
$$

Since $R_{k}$ converges uniformly to $R$ and the latter preserves the norm of the first $n$ components, we know that, for $k$ large, $\left\|L^{k}\right\|_{\infty}<2 T \sqrt{n \mu}$. Moreover, $R_{k}(t, 0) \bar{\Pi}_{N}\left(\psi_{0}\right)$ converges uniformly to $R(t, 0) \bar{\Pi}_{N}\left(\psi_{0}\right)$. In particular, according to the definition of $R, \bar{\Pi}_{n}^{N}\left(R_{k}(t, 0) \bar{\Pi}_{N}\left(\psi_{0}\right)\right)$ converges uniformly to the solution of $\left(\Theta_{n}\right)$ corresponding to the control $u$ and starting from $\bar{\Pi}_{n}\left(\psi_{0}\right)=\xi_{0}$.

Since $u$ drives system $\left(\Sigma_{n}\right)$ from $\xi_{0} /\left\|\xi_{0}\right\|$ to $\xi_{1} /\left\|\xi_{1}\right\|$, then it steers system $\left(\Theta_{n}\right)$ from $\xi_{0}$ to $e^{-v(T) A^{(n)}} \xi_{1}\left(\left\|\xi_{0}\right\| /\left\|\xi_{1}\right\|\right)$. Therefore,

$$
\left\|P^{k}(T)-e^{-v(T) A^{(n)}} \xi_{1} \frac{\left\|\xi_{0}\right\|}{\left\|\xi_{1}\right\|}\right\|<3 T \sqrt{n \mu},
$$

if $k$ is large enough. Let us fix $\mu$ small enough in order to have

$$
3 T \sqrt{n \mu}<\varepsilon .
$$

Then,

$$
\begin{aligned}
\left\|\Pi_{n}\left(e^{-v_{k}(T) A} \psi^{k}(T)\right)-\Pi_{n}\left(e^{-v(T) A} \psi_{1}\right)\right\| \leqslant & \left\|\Pi_{n}\left(e^{-v_{k}(T) A} \psi^{k}(T)\right)-\Pi_{n}\left(e^{-v(T) A} \psi_{1}\right) \frac{\left\|\xi_{0}\right\|}{\left\|\xi_{1}\right\|}\right\| \\
& +\left\|\Pi_{n}\left(e^{-v(T) A} \psi_{1}\right) \frac{\left\|\xi_{0}\right\|}{\left\|\xi_{1}\right\|}-\Pi_{n}\left(e^{-v(T) A} \psi_{1}\right)\right\| \\
& =\left\|P^{k}(T)-e^{-v(T) A^{(n)}} \xi_{1} \frac{\left\|\xi_{0}\right\|}{\left\|\xi_{1}\right\|}\right\|+\left\|\Pi_{n}\left(e^{-v(T) A} \psi_{1}\right)\right\| \frac{\left|\left\|\xi_{1}\right\|-\left\|\xi_{0}\right\|\right|}{\left\|\xi_{1}\right\|} \\
& <3 T \sqrt{n \mu}+\left|\left\|\xi_{1}\right\|-\left\|\xi_{0}\right\|\right| \\
& <2 \varepsilon,
\end{aligned}
$$

provided that $k$ is large enough.

As a consequence of Proposition 4.4, for $k$ large enough the moduli of the first $n$ components of $\psi^{k}(T)$ are close to those of the first $n$ components of $\psi_{1}$. The proposition below will be used to show that their phases can also be made as close as required by applying a suitable control on an arbitrarily small time interval.

Proposition 4.5. For every $\tilde{\varepsilon}>0$, every $\tilde{\lambda}_{1}, \ldots, \tilde{\lambda}_{n} \in \mathbf{R}$, and every $s_{1} \in \mathbf{R}$ there exist $s_{2}>s_{1}$ and $w \in \mathbf{R}^{n}$ with $\|w\| \leqslant \tilde{\varepsilon}$ such that $\tilde{\lambda}_{i} s_{2} \equiv w_{i} \bmod 2 \pi$ for every $i=1, \ldots, n$. As a consequence, given a skew-adjoint discrete-spectrum control system $(\tilde{A}, \tilde{B},(0, \tilde{\delta}))$, for every $v_{1} \in \mathbf{R}$ and every $\tau>0$ small enough there exists a constant control function $\tilde{u}:[0, \tau] \rightarrow(\tilde{\delta},+\infty)$ such that every trajectory $\tilde{\psi}(\cdot)$ of $\dot{\psi}=u \tilde{A} \psi+\tilde{B} \psi$ corresponding to $\tilde{u}(\cdot)$ satisfies $\| \Pi_{n}(\tilde{\psi}(\tau))-$ $\Pi_{n}\left(e^{v_{1} \tilde{A}} \tilde{\psi}(0)\right) \| \leqslant \tilde{\varepsilon}$ (where $\Pi_{n}$ denotes the orthogonal projection on the space spanned by the first $n$ eigenvectors of $\tilde{A})$. 
Proof. The first part of the statement is a simple application of the Poincaré recurrence theorem. Indeed, since the dynamics $s \mapsto x_{0}+s\left(\tilde{\lambda}_{1}, \ldots, \tilde{\lambda}_{n}\right)$ on the $n$-dimensional torus preserve volumes and distances, then the constant vector field $\left(\tilde{\lambda}_{1}, \ldots, \tilde{\lambda}_{n}\right)$ is recurrent at every point of the torus, and in particular at the origin $x_{0}=0$. Therefore any neighborhood $\mathcal{N}$ of the origin is sent, after a suitably long time (which can be assumed to be larger than $s_{1}$ ), to another neighborhood of the origin isometric to $\mathcal{N}$. Taking $\mathcal{N}$ equal to the ball of radius $\tilde{\varepsilon}$ centered at the origin, the first part of the claim is proven.

In order to conclude the proof, fix a piecewise constant control function $\tilde{u}:[0, \tau] \rightarrow(\tilde{\delta},+\infty)$ and a solution $\tilde{\psi}$ of $\dot{\psi}=u \tilde{A} \psi+\tilde{B} \psi$ corresponding to $\tilde{u}$. Set

$$
\tilde{q}(t)=e^{-\int_{0}^{t} \tilde{u}(s) d s \tilde{A}} \tilde{\psi}(t)
$$

and notice that, according to $(2.3),\left|\dot{\tilde{q}}_{j}(t)\right| \leqslant\left\|\tilde{B} \tilde{\phi}_{j}\right\|$ for every $j \in \mathbf{N}$, where $\tilde{\phi}_{j}$ denotes the $j$-th eigenvector of $\tilde{A}$ and $\tilde{q}_{j}=\left\langle\tilde{q}, \tilde{\phi}_{j}\right\rangle$. Therefore, $\left\|\Pi_{n}(\tilde{q}(\tau))-\Pi_{n}(\tilde{q}(0))\right\| \leqslant C \tau$ for some positive constant $C$ independent of $\tilde{u}$ and of $\tilde{\psi}$. Then

$$
\begin{aligned}
\left\|\Pi_{n}(\tilde{\psi}(\tau))-\Pi_{n}\left(e^{v_{1} \tilde{A}} \tilde{\psi}(0)\right)\right\| & =\left\|\Pi_{n}\left(e^{-v_{1} \tilde{A}} \tilde{\psi}(\tau)\right)-\Pi_{n}(\tilde{\psi}(0))\right\| \\
& =\left\|\Pi_{n}\left(e^{\left(\int_{0}^{\tau} \tilde{u}(t) d t-v_{1}\right) \tilde{A}} \tilde{q}(\tau)\right)-\Pi_{n}(\tilde{q}(0))\right\| \\
& \leqslant\left\|\Pi_{n}\left(e^{\left(\int_{0}^{\tau} \tilde{u}(t) d t-v_{1}\right) \tilde{A}} \tilde{q}(\tau)\right)-\Pi_{n}(\tilde{q}(\tau))\right\|+C \tau .
\end{aligned}
$$

Fix $\tau<\tilde{\varepsilon} /(2 C)$ so that

$$
\left\|\Pi_{n}(\tilde{\psi}(\tau))-\Pi_{n}\left(e^{v_{1} \tilde{A}} \tilde{\psi}(0)\right)\right\| \leqslant\left\|\Pi_{n}\left(e^{\left(\int_{0}^{\tau} \tilde{u}(t) d t-v_{1}\right) \tilde{A}} \tilde{q}(\tau)\right)-\Pi_{n}(\tilde{q}(\tau))\right\|+\frac{\tilde{\varepsilon}}{2} .
$$

Notice that

$$
\Pi_{n}\left(e^{\left(\int_{0}^{\tau} \tilde{u}(t) d t-v_{1}\right) \tilde{A}} \tilde{q}(\tau)\right)=\operatorname{diag}\left(e^{i \tilde{\lambda}_{1}\left(\int_{0}^{\tau} \tilde{u}(t) d t-v_{1}\right)}, \ldots, e^{i \tilde{\lambda}_{n}\left(\int_{0}^{\tau} \tilde{u}(t) d t-v_{1}\right)}\right) \Pi_{n}(\tilde{q}(\tau)) .
$$

The first part of the claim ensures the existence of $v_{2}$ arbitrarily large such that if

$$
\int_{0}^{\tau} \tilde{u}(t) d t-v_{1}=v_{2}
$$

then the norm of the matrix

$$
\operatorname{diag}\left(e^{i \tilde{\lambda}_{1}\left(\int_{0}^{\tau} \tilde{u}(t) d t-v_{1}\right)}, \ldots, e^{i \tilde{\lambda}_{n}\left(\int_{0}^{\tau} \tilde{u}(t) d t-v_{1}\right)}\right)-\operatorname{Id}_{n}
$$

is smaller than $\tilde{\varepsilon} / 2$. Take $v_{2}$ large enough to satisfy $\left(v_{1}+v_{2}\right) / \tau>\tilde{\delta}$. Then $\tilde{u} \equiv\left(v_{1}+v_{2}\right) / \tau$ satisfies (4.12) and, because of (4.11),

$$
\left\|\Pi_{n}(\tilde{\psi}(\tau))-\Pi_{n}\left(e^{v_{1} \tilde{A}} \tilde{\psi}(0)\right)\right\| \leqslant \tilde{\varepsilon},
$$

independently of $\tilde{\psi}$.

To conclude the proof of Theorem 2.4 we extend the interval of definition of the control function $u_{k}$ introduced above by taking $u_{k}(T+t)=\tilde{u}(t)$ for $t \in[0, \tau]$ where $\tilde{u}$ is the control obtained by applying Proposition 4.5 with $v_{1}=v(T)-v_{k}(T), \tilde{A}=A, \tilde{B}=B, \tilde{\varepsilon}=\varepsilon$. Then, for $k$ large enough, the corresponding trajectory $\psi^{k}:[0, T+\tau] \rightarrow \mathcal{S}$ satisfies $\left\|\Pi_{n}\left(\psi^{k}(T+\tau)\right)-\Pi_{n}\left(\psi_{1}\right)\right\|<3 \varepsilon$ and therefore, due to (4.5),

$$
\left\|\Pi_{n}\left(\psi^{k}(T+\tau)\right)-\psi_{1}\right\|<4 \varepsilon
$$

It is now enough to show that $\left\|\psi^{k}(T+\tau)-\Pi_{n}\left(\psi^{k}(T+\tau)\right)\right\|$ can be made arbitrarily small by choosing a suitably small $\varepsilon$. To this aim we notice that the inequality $\left\|\Pi_{n}\left(\psi^{k}(T+\tau)\right)\right\|>1-4 \varepsilon$ implies, for $\varepsilon<1 / 4$, that $\| \psi^{k}(T+\tau)-$ $\Pi_{n}\left(\psi^{k}(T+\tau)\right) \|^{2}<1-(1-4 \varepsilon)^{2}=8 \varepsilon-16 \varepsilon^{2}$ and this concludes the proof of Theorem 2.4. 


\subsection{Lower bound on the steering time}

In this section we prove a lower bound on the steering time for a skew-adjoint discrete-spectrum control system without assuming that it satisfies the hypotheses of Theorem 2.4 nor any other controllability assumption.

Proposition 4.6. Let $(A, B,(0, \delta))$ be a skew-adjoint discrete-spectrum control system. Fix $\psi_{0}, \psi_{1}$ in $\mathcal{S}$ and $\varepsilon>0$. Then if a piecewise constant control $u:\left[0, T_{u}\right] \rightarrow(0, \delta)$ steers system (2.1) from $\psi_{0}$ to an $\varepsilon$-neighborhood of $\psi_{1}$, then

$$
T_{u} \geqslant \frac{1}{\delta} \sup _{k \in \mathbf{N}} \frac{||\left\langle\phi_{k}, \psi_{0}\right\rangle|-|\left\langle\phi_{k}, \psi_{1}\right\rangle||-\epsilon}{\left\|B \phi_{k}\right\|}
$$

where $\left(\phi_{k}\right)_{k \in \mathbf{N}}$ denotes the orthonormal basis of eigenvectors of $A$.

Proof. Fix an initial condition $\psi_{0}$ in $\mathcal{S}$, a piecewise constant control $u:\left[0, T_{u}\right] \rightarrow(0, \delta)$, and denote by $\psi^{u}:\left[0, T_{u}\right] \rightarrow$ $\mathscr{H}$ the corresponding solution of the system (2.1) satisfying $\psi^{u}(0)=\psi_{0}$.

Write $u$ as $u(t)=\sum_{j=0}^{n} u_{j} \chi_{\left[t_{j}, t_{j+1}\right)}(t)$ where $0=t_{1}<t_{2}<\cdots<t_{n+1}=T_{u}$ and $u_{1}, \ldots, u_{n}$ belong to $(0, \delta)$. In the spirit of Section 4.1, associate to $u$ the piecewise constant control $v:\left[0, T_{v}\right] \rightarrow \mathbf{R}$ given by $v(t)=\sum_{j=0}^{n} v_{j} \chi_{\left[\tau_{j}, \tau_{j+1}\right)}(t)$ with $v_{j}=1 / u_{j}$ for all $j=1, \ldots, n$ and $\tau_{j}$ defined by induction as $\tau_{1}=0, \tau_{j+1}=\tau_{j}+\left(t_{j+1}-t_{j}\right) u_{j}$ for $j \geqslant 1$.

Define $\psi^{\nu}:\left[0, T_{\nu}\right] \rightarrow \mathscr{H}$ as the solution of system (4.2) corresponding to $v$ and satisfying $\psi^{\nu}(0)=\psi_{0}$. Define by $m_{k}=\left|\left\langle\psi^{v}, \phi_{k}\right\rangle\right|$ the modulus of the $k^{\text {th }}$ coordinate of $\psi^{v}$.

By definition $m_{k}$ is absolutely continuous and Eq. (4.10) implies that

$$
\dot{m}_{k} \leqslant \sum_{j=1}^{\infty}\left|b_{j k}\right| m_{j} \leqslant\left(\sum_{j=1}^{\infty}\left|\left\langle B \phi_{j}, \phi_{k}\right\rangle\right|^{2}\right)^{1 / 2}=\left\|B \phi_{k}\right\| .
$$

Applying the mean value theorem, one gets

$$
\left\|\left\langle\psi^{v}(0), \phi_{k}\right\rangle|-|\left\langle\psi^{v}\left(T_{v}\right), \phi_{k}\right\rangle|| \leqslant T_{v}\right\| B \phi_{k} \| .
$$

Notice that $T_{\nu}=\sum_{j=1}^{n}\left(t_{j+1}-t_{j}\right) u_{j} \leqslant\left(t_{n}-t_{1}\right) \delta=T_{u} \delta$, that is,

$$
T_{u} \geqslant \frac{1}{\delta} T_{\nu}
$$

Since, by assumption, $\left|\psi^{v}\left(T_{v}\right)-\psi_{1}\right|<\epsilon$, then (4.14) implies

$$
T_{\nu} \geqslant \sup _{k \in \mathbf{N}} \frac{\|\left\langle\phi_{k}, \psi_{0}\right\rangle|-|\left\langle\phi_{k}, \psi_{1}\right\rangle||-\epsilon}{\left\|B \phi_{k}\right\|} .
$$

Plugging this last inequality into (4.15), we obtain (4.13).

We insist on the fact that this result is valid whenever system (2.1) is or is not approximately controllable.

Remark 4.7. When $B$ is bounded, the same estimate as above is valid for other classes of controls (not only piecewise constant functions but also measurable bounded or locally integrable) as soon as we can define a unique solution of system (2.1) that satisfies (2.3). See Remark 2.2.

Remark 4.8. It follows from (4.13) that, in general, approximate controllability does not imply finite-time approximate controllability. Indeed, if $B \phi_{k}$ tends to 0 as $k$ goes to infinity, then for every $T>0$ the attainable set at time $T$ from a given point $\psi_{0}$ is not dense in $\mathcal{S}$ since for every $\varepsilon \in(0,1)$, for $k$ large enough, $\phi_{k}$ is not $\varepsilon$-approximately attainable from $\psi_{0}$ in time $T$.

\section{Controllability for density matrices}

\subsection{Physical motivations}

A density matrix (sometimes called density operator) is a non-negative, self-adjoint operator of trace class [32, Vol. I] on a Hilbert space. The trace of a density matrix is normalized to one. As a consequence of the defi- 
nition a density matrix is a compact operator (hence with discrete spectrum) and can always be written as a weighted sum of projectors,

$$
\rho=\sum_{j=1}^{\infty} P_{j} \varphi_{j} \varphi_{j}^{*},
$$

where $P_{j} \in[0,1], \sum_{j} P_{j}=1$, and $\varphi_{j} \varphi_{j}^{*}$ is the orthogonal projector on the space spanned by $\varphi_{j}$ with $\varphi_{j}^{*}(\cdot)=\left\langle\varphi_{j}, \cdot\right\rangle$. Here $\left\{\varphi_{j}\right\}_{j \in \mathbf{N}}$ is a set of normalized vectors not necessarily orthogonal.

The density matrix is used to describe the evolution of systems whose initial wave function is not known precisely, but only with a certain probability, or when one is dealing with an ensemble of identical systems that cannot be prepared precisely in the same state. More precisely (5.1) describes a system whose state is known to be $\varphi_{j}$ with probability $P_{j}, j \in \mathbf{N}$. Given an observable $A$ (i.e. a self-adjoint operator, for instance the drift Hamiltonian) the mean value of $A$ is $\operatorname{Tr}(\rho A)=\sum_{j=1}^{\infty} P_{j}\left\langle\varphi_{j}, A \varphi_{j}\right\rangle$, where $\left\langle\varphi_{j}, A \varphi_{j}\right\rangle$ represents the mean value of the observable $A$ in the state $\varphi_{j}$. When for some $k \in \mathbf{N}$ we have $P_{k}=1$ and $P_{j}=0$ for every $j \neq k$, one says that $\rho$ describes a pure state, otherwise one says that $\rho$ describes a mixed state. In the case of pure states, the physical description via the density matrix is equivalent to the one via the wave function. Notice that for a pure state $\operatorname{Tr}\left(\rho^{2}\right)=1$ while for a mixed state one has $\operatorname{Tr}\left(\rho^{2}\right)<1$.

Without loss of generality it is possible to require that $\left\{\varphi_{j}\right\}_{j \in \mathbf{N}}$ is an orthonormal basis (i.e. a basis of normalized eigenvectors of $\rho$ ). In this case $\left\{P_{j}\right\}_{j \in \mathbf{N}}$ is the spectrum of $\rho$.

The time evolution of the density matrix is determined by the evolutions of the states $\varphi_{j}$, namely

$$
\rho(t)=\mathbf{U}(t) \rho(0) \mathbf{U}^{*}(t)
$$

where $\mathbf{U}(t)$ is the operator of temporal evolution (the resolvent) and $\mathbf{U}^{*}(t)$ its adjoint. Notice that the spectrum of $\rho(t)$ is constant along the motion.

\subsection{Statement of the result}

Fix $\delta>0$ and let $(A, B,(0, \delta))$ be a skew-adjoint discrete-spectrum control system on a Hilbert space $\mathscr{H},\left(\varphi_{j}\right)_{j \in \mathbf{N}}$ an orthonormal basis of $\mathscr{H}$ (not necessarily of eigenvectors of $A$ ), $\left\{P_{j}\right\}_{j \in N}$ a sequence of non-negative numbers such that $\sum_{j=1}^{\infty} P_{j}=1$, and denote by $\rho$ the density matrix

$$
\rho=\sum_{j=1}^{\infty} P_{j} \varphi_{j} \varphi_{j}{ }^{*} .
$$

Definition 5.1. Two density matrices $\rho_{0}$ and $\rho_{1}$ are said to be unitarily equivalent if there exists a unitary transformation $\mathbf{U}$ of $\mathscr{H}$ such that $\rho_{1}=\mathbf{U} \rho_{0} \mathbf{U}^{*}$.

Obviously the controllability question for the evolution of the density matrix makes sense only for pairs $\left(\rho_{0}, \rho_{1}\right)$ of initial and final density matrices that are unitarily equivalent. Notice that this is a quite strong assumption, since it implies that the eigenvalues of $\rho_{0}$ and $\rho_{1}$ are the same. Controllability results in the case of density matrices that are not unitarily equivalent have been obtained in the case of open systems (i.e. systems evolving under a suitable non-unitary evolution) in the finite-dimensional case. See for instance [9].

Next section is devoted to the proof of the following theorem.

Theorem 5.2. Let $\rho_{0}$ and $\rho_{1}$ be two unitarily equivalent density matrices. Then, under the hypotheses of Theorem 2.4, for every $\varepsilon>0$ there exists a piecewise constant control steering the density matrix from $\rho_{0} \varepsilon$-approximately to $\rho_{1}$ i.e. there exist $k \in \mathbf{N}, t_{1}, \ldots, t_{k}>0$ and $u_{1}, \ldots, u_{k} \in(0, \delta)$ such that setting $\mathbf{V}=e^{t_{k}\left(A+u_{k} B\right)} \cdots e^{t_{1}\left(A+u_{1} B\right)}$, one has $\left\|\rho_{1}-\mathbf{V} \rho_{0} \mathbf{V}^{*}\right\|<\varepsilon$, where $\|\cdot\|$ denotes the operator norm on $\mathscr{H}$.

Remark 5.3. As Theorem 3.4 is a particularization of Theorem 2.4 to the controlled Schrödinger equation, the hypotheses of Theorem 3.4 imply $\varepsilon$-approximate controllability of the corresponding density matrix. 


\subsection{Proof of Theorem 5.2}

The proof uses the notations of Section 4. As noticed in Section 4.1, the theorem can be restated in terms of the evolution of the density matrix corresponding to the control system $\dot{\psi}=(u A+B) \psi, u \in(\delta,+\infty)$.

Fix $\rho_{0}$ and $\rho_{1}$ unitarily equivalent and let $\mathbf{U}$ be such that $\rho_{1}=\mathbf{U} \rho_{0} \mathbf{U}^{*}$. Write

$$
\rho_{0}=\sum_{j=1}^{\infty} P_{j} \varphi_{0, j} \varphi_{0, j}{ }^{*},
$$

with $\left(P_{j}\right)_{j \in N}$ a sequence of non-negative numbers whose sum is one, and $\left(\varphi_{0, j}\right)_{j \in N}$ an orthonormal basis of $\mathscr{H}$. Then

$$
\rho_{1}=\sum_{j=1}^{\infty} P_{j} \varphi_{1, j} \varphi_{1, j}{ }^{*},
$$

with $\varphi_{1, j}=\mathbf{U} \varphi_{0, j}$ for every $j \in \mathbf{N}$.

Choose $\varepsilon>0$. Let $m \in \mathbf{N}$ be such that

$$
\sum_{j>m} P_{j}<\varepsilon
$$

The idea is to follow the strategy applied in the proof of Theorem 2.4 in order to simultaneously approximately steer $m$ copies of system $(A, B,(0, \delta))$ from $\varphi_{0, j}$ to $\varphi_{1, j}, j=1, \ldots, m$.

Let $\eta>0$ be a small constant depending on $m$ and $\varepsilon$, to be fixed later. There exists $n=n(\eta)>m$ such that, for every $j=1, \ldots, m$ and for $k=0,1$,

$$
\left\|\varphi_{k, j}-\Pi_{n} \varphi_{k, j}\right\|<\eta .
$$

By construction, when $\eta$ gets small, the two families $\left(\Pi_{n} \varphi_{k, j}\right)_{j=1}^{m}, k=0,1$, tend to two orthonormal families. Hence, there exists a matrix $M$ in $\mathrm{SU}(n)$ such that

$$
\left\|M\left(\bar{\Pi}_{n} \varphi_{0, j}\right)-\bar{\Pi}_{n} \varphi_{1, j}\right\|<\varepsilon
$$

for $j=1, \ldots, m$ provided that $\eta$ is small enough (and, consequently, $n$ is large enough).

Without loss of generality we may assume that $B^{(n)}$ is connected. Claim 4.1 can be extended to the following result.

\section{Claim 5.4. The control system}

$$
\dot{g}=\left(u A^{(n)}+B^{(n)}\right) g, \quad g \in U(n),
$$

is controllable in the following sense: for any $g_{0}, g_{1}$ in $U(n)$, there exists a unitary complex number $e^{i \theta}$ with $0 \leqslant \theta \leqslant$ $2 \pi / n$, a time $T>0$ and a piecewise constant function $u:[0, T] \rightarrow(\delta,+\infty)$ such that the solution $g^{u}:[0, T] \rightarrow U(n)$ of (5.4) with initial condition $g^{u}(0)=g_{0}$ satisfies $e^{i \theta} g^{u}(T)=g_{1}$.

Proof. Let us first assume that at least one among $A^{(n)}$ and $B^{(n)}$ has non-zero trace and hence does not belong to $\mathfrak{s u}(n)$. In this case the inclusion (4.3), with $A^{(n)}=\mathfrak{A}$ and $B^{(n)}=\mathfrak{B}$, implies that $\operatorname{Lie}\left(A^{(n)}, B^{(n)}\right)=\mathfrak{u}(n)$. Classical controllability results for right invariant systems on compact Lie groups (see $[24,37]$ ) ensure that the attainable set from $g_{0}$ of (5.4) coincides with $U(n)$ so that the claim holds with $\theta=0$.

It remains to consider the case in which the traces of $A^{(n)}$ and $B^{(n)}$ are zero, i.e. $A^{(n)}$ and $B^{(n)}$ belong to $\mathfrak{s u}(n)$. In this case (4.3) implies that $\operatorname{Lie}\left(A^{(n)}, B^{(n)}\right)=\mathfrak{s u}(n)$, and therefore the attainable set from $g_{0}$ of (5.4) coincides with $g_{0} \mathrm{SU}(n)$, the set of matrices of $U(n)$ having the same determinant as $g_{0}$. Given a target $g_{1}$ there exists $\vartheta \in[0,2 \pi]$ such that $\operatorname{det}\left(g_{0}\right)=e^{-i \vartheta} \operatorname{det}\left(g_{1}\right)=\operatorname{det}\left(e^{-i \frac{\vartheta}{n}} g_{1}\right)$. Hence the claim holds true with $\theta=\vartheta / n$.

Let $T>0, u:[0, T] \rightarrow(\delta,+\infty)$ and $0 \leqslant \theta \leqslant 2 \pi / n$ be such that the control $u$ steers system (5.4) from $I_{n}$ to $e^{i \theta} M$. Notice that, without loss of generality, $2 \pi / n<\varepsilon$. 
Let $\mu>0$ be a small constant to be fixed later. Fix $N \in \mathbf{N}$ such that

$$
\left\|\left(b_{j l}\right)_{l>N}\right\|_{l^{2}}<\mu
$$

for every $j=1, \ldots, n$. Let us apply Claim 4.3 to the control function $u$ and denote by $\left(u_{k}\right)_{k \in \mathbf{N}}$ the sequence of piecewise constant control functions obtained in this way. Write, moreover, $v(t)=\int_{0}^{t} u(\tau) d \tau$ and $v_{k}(t)=\int_{0}^{t} u_{k}(\tau) d \tau$. For every $k \in \mathbf{N}$ write $u_{k}$ as

$$
u_{k}(t)=\sum_{j=1}^{p_{k}} w_{j}^{k} \chi_{\left[t_{j}^{k}, t_{j+1}^{k}\right)}(t), \quad t \in[0, T],
$$

with $0=t_{1}^{k} \leqslant \cdots \leqslant t_{p_{k}}^{k}=T$ and denote by $\mathbf{V}_{k}$ the unitary transformation

$$
\mathbf{V}_{k}=e^{\left(t_{p_{k}}^{k}-t_{p_{k}-1}^{k}\right)\left(w_{p_{k}-1}^{k} A+B\right)} \cdots e^{\left(t_{2}^{k}-t_{1}^{k}\right)\left(w_{1}^{k} A+B\right)} .
$$

For every $j=1, \ldots, m$,

$$
\begin{aligned}
\left\|\Pi_{n}\left(e^{\left(v(T)-v^{k}(T)\right) A} \mathbf{V}_{k} \varphi_{0, j}\right)-\Pi_{n}\left(\varphi_{1, j}\right)\right\| \leqslant & \left\|\bar{\Pi}_{n}\left(e^{\left(v(T)-v^{k}(T)\right) A} \mathbf{V}_{k} \varphi_{0, j}\right)-e^{i \theta} M\left(\bar{\Pi}_{n} \varphi_{0, j}\right)\right\| \\
& +\left\|e^{i \theta} M\left(\bar{\Pi}_{n} \varphi_{0, j}\right)-M\left(\bar{\Pi}_{n} \varphi_{0, j}\right)\right\|+\left\|M\left(\bar{\Pi}_{n} \varphi_{0, j}\right)-\bar{\Pi}_{n}\left(\varphi_{1, j}\right)\right\| .
\end{aligned}
$$

The same computations as in Section 4.4 (cf. (4.9)) show that, for every $j=1, \ldots, m$,

$$
\left\|\bar{\Pi}_{n}\left(e^{\left(v(T)-v^{k}(T)\right) A} \mathbf{V}_{k} \varphi_{0, j}\right)-e^{i \theta} M\left(\bar{\Pi}_{n} \varphi_{0, j}\right)\right\| \leqslant 2 \varepsilon
$$

for $\mu$ small and $k$ large enough. Since $0 \leqslant \theta \leqslant 2 \pi / n<\varepsilon$, then, for every $j=1, \ldots, m$,

$$
\left\|e^{i \theta} M\left(\bar{\Pi}_{n} \varphi_{0, j}\right)-M\left(\bar{\Pi}_{n} \varphi_{0, j}\right)\right\| \leqslant\left|e^{i \theta}-1\right|<\varepsilon .
$$

Hence, because of (5.3), for $k$ large enough, for every $j=1, \ldots, m$,

$$
\left\|\Pi_{n}\left(e^{\left(v(T)-v^{k}(T)\right) A} \mathbf{V}_{k} \varphi_{0, j}\right)-\Pi_{n}\left(\varphi_{1, j}\right)\right\|<4 \varepsilon .
$$

Applying Proposition 4.5 we can, up to the extension of $u^{k}$ to a piecewise constant control defined on a larger interval, assume that

$$
\left\|\Pi_{n}\left(\mathbf{V}_{k} \varphi_{0, j}\right)-\Pi_{n}\left(\varphi_{1, j}\right)\right\|<5 \varepsilon,
$$

for every $j=1, \ldots, m$. Therefore,

$$
\begin{aligned}
\left\|\mathbf{V}_{k} \rho_{0} \mathbf{V}_{k}^{*}-\rho_{1}\right\| & =\left\|\sum_{j=1}^{\infty} P_{j}\left(\left(\mathbf{V}_{k} \varphi_{0, j}\right)\left(\mathbf{V}_{k} \varphi_{0, j}\right)^{*}-\varphi_{1, j} \varphi_{1, j}^{*}\right)\right\| \\
& \leqslant\left\|\sum_{j=1}^{m} P_{j}\left(\left(\mathbf{V}_{k} \varphi_{0, j}\right)\left(\mathbf{V}_{k} \varphi_{0, j}\right)^{*}-\varphi_{1, j} \varphi_{1, j}^{*}\right)\right\|+2 \varepsilon \\
& \leqslant \sum_{j=1}^{m} P_{j}\left(\left\|\mathbf{V}_{k} \varphi_{0, j}\right\|+\left\|\varphi_{1, j}\right\|\right)\left\|\mathbf{V}_{k} \varphi_{0, j}-\varphi_{1, j}\right\|+2 \varepsilon \\
& \leqslant 2(5 \varepsilon)+2 \varepsilon=12 \varepsilon,
\end{aligned}
$$

provided that $k$ is large enough. This concludes the proof of Theorem 5.2.

\section{Examples}

\subsection{Perturbation of the spectrum}

The scope of Section 6 is to show how the general controllability results obtained in the previous sections can be applied in specific cases. In particular, we want to show how the conditions on the spectrum of the Schrödinger operator appearing in the hypotheses of Theorem 3.4 can be checked in practice. 
Let us adopt the notations of Section 3 for the domain $\Omega$, the wave function $\psi$, and the uncontrolled and controlled potentials $V$ and $W$. Throughout this section we assume that one of the hypotheses (i) or (ii) of Corollary 3.3 holds true. Thus, $(A, B, U)$ is a well-defined controlled Schrödinger equation, where $A=-i(-\Delta+V)$ and $B=-i W$.

The study of the examples below is based on the simple idea that, even if the hypotheses of Theorem 3.4 are not satisfied by the operators $A$ and $B$, one can anyway ensure that they hold true for $A_{\mu}=-i(-\Delta+V+\mu W)$ and $B_{\mu}=-i W$ for some $\mu$ in the interior of $U$. This is enough to conclude that the system $\dot{\psi}=A \psi+u B \psi, u \in U$, is approximately controllable, since the replacement of $(A, B)$ by $\left(A_{\mu}, B_{\mu}\right)$ corresponds to a reparameterization of $U$ that sends $u$ into a new control $u-\mu \in U-\mu$ and $V$ into $V+\mu W$. Although the spectrum of $A_{\mu}$ is not in general explicitly computable, we can nevertheless deduce some crucial properties about it by applying standard perturbation arguments. Theorem 6.1 recalls, in a simplified version suitable for our purposes, some classical perturbation results describing the dependence on $\mu$ of the spectrum of $-\Delta+V+\mu W$. (See [25, Chapter VII, Remark 4.22], [33, §II.10, Theorem 1] and also [6].)

Theorem 6.1. Let $U$ be an open interval containing zero. Assume either that (i) $\Omega$ is bounded, $V$, $W$ belong to $L^{\infty}(\Omega)$ or that (ii) $\Omega=\mathbf{R}^{d}, V$ belongs to $L_{\text {loc }}^{1}\left(\mathbf{R}^{d}\right)$, W belongs to $L^{\infty}\left(\mathbf{R}^{d}\right), \lim _{\|x\| \rightarrow+\infty} V(x)=+\infty$ and $\inf _{x \in \mathbf{R}^{d}} V(x)>$ $-\infty$. In both cases (i) and (ii) assume that each eigenvalue of the Schrödinger operator $-\Delta+V$ is simple. Denote by $\left(\lambda_{k}\right)_{k \in \mathbf{N}}$ the sequence of eigenvalues of $-\Delta+V$ and by $\left(\phi_{k}\right)_{k \in \mathbf{N}}$ the corresponding eigenfunctions. Then, for any $k$ in $\mathbf{N}$, there exist two analytic curves $\Lambda_{k}: U \rightarrow \mathbf{C}$ and $\Phi_{k}: U \rightarrow L^{2}(\Omega)$ such that:

- $\Lambda_{k}(0)=\lambda_{k}$ and $\Phi_{k}(0)=\phi_{k}$;

- for any $\mu$ in $U,\left(\Lambda_{k}(\mu)\right)_{k \in \mathbf{N}}$ is the family of eigenvalues of $\Delta-V+\mu W$ counted according to their multiplicities and $\left(\Phi_{k}(\mu)\right)_{k \in \mathbf{N}}$ is an orthonormal basis of corresponding eigenfunctions;

- $\Lambda_{k}^{\prime}(0)=\int_{\Omega} W(x)\left|\phi_{k}(x)\right|^{2} d x$.

We check below that if the derivatives $\Lambda_{k}^{\prime}(0)$ are Q-linearly independent then for almost every $\mu \in U$ the eigenvalues of $-\Delta+V+\mu W$ are $\mathbf{Q}$-linearly independent. This fact is used in the following to apply Theorem 3.4 to situations in which the uncontrolled Schrödinger operator has a resonant spectrum.

Recall that, in the notations of Section 4, for any pair of integers $j, k \in \mathbf{N}$,

$$
b_{j k}=\int_{\Omega} W(x) \phi_{j}(x) \phi_{k}(x) d x .
$$

In particular, $\Lambda_{k}^{\prime}(0)=\int_{\Omega} W(x)\left|\phi_{k}(x)\right|^{2} d x$ is equal to $b_{k k}$.

Proposition 6.2. Let $U$ be an open interval containing zero and assume that $\Omega, V$ and $W$ satisfy one of the hypotheses (i) or (ii) of Theorem 6.1 and that the eigenvalues of $-\Delta+V$ are simple. If the elements of the sequence $\left(b_{k k}\right)_{k \in \mathbf{N}}$ are $\mathbf{Q}$-linearly independent, then for almost every $\mu$ in $U$ the elements of $\left(\Lambda_{k}(\mu)\right)_{k \in \mathbf{N}}$ are $\mathbf{Q}$-linearly independent.

Proof. Let $l \in \mathbf{N}$ and $z=\left(z_{1}, \ldots, z_{l}\right) \in \mathbf{Q}^{l}$. Denote by $\Upsilon_{z}$ the subset of elements $\mu$ in $U$ such that $\sum_{j=1}^{l} z_{j} \Lambda_{j}(\mu)=0$. Since each $\mu \mapsto \Lambda_{k}(\mu)$ is an analytic function, then $\Upsilon_{z}$ is either equal to $U$ or to a countable subset of $U$. Since $b_{11}=\Lambda_{1}^{\prime}(0), \ldots, b_{l l}=\Lambda_{l}^{\prime}(0)$ are Q-linearly independent, then $\Upsilon_{z}=U$ if and only if $z=0$. Hence, the union $\boldsymbol{\Upsilon}=$ $\bigcup_{l \in \mathbf{N}} \bigcup_{z \in \mathbf{Q}^{l}, z \neq 0} \Upsilon_{z}$ has Lebesgue measure zero, since it is countable. By construction, if $\mu$ does not belong to $\boldsymbol{\Upsilon}$, the elements of $\left(\Lambda_{k}(\mu)\right)_{k \in \mathbf{N}}$ are $\mathbf{Q}$-linearly independent.

The other crucial hypothesis of Theorem 3.4 is that $b_{j, j+1} \neq 0$ for every $j \in \mathbf{N}$ (or, more generally, that $B^{(n)}=$ $\left(b_{j k}\right)_{j, k=1}^{n}$ is frequently connected, see Remark 4.2). By the same analyticity argument as above one checks that either such hypothesis is always false or it is true for almost every $\mu \in U$.

Corollary 6.3. Let $U$ be an open interval containing zero and assume that $\Omega, V$ and $W$ satisfy one of the hypotheses (i) or (ii) of Theorem 6.1 and that the eigenvalues of $-\Delta+V$ are simple. Assume moreover that the elements of the sequence $\left(b_{k k}\right)_{k \in \mathbf{N}}$ are $\mathbf{Q}$-linearly independent and that $B^{(n)}$ is frequently connected. Then the controlled Schrödinger equation associated with $\Omega, V, W$ and $\tilde{U}$ is approximately controllable for every $\tilde{U} \subset U$ with non-empty interior. 


\subsection{D harmonic oscillator}

In this section we study the Schrödinger equation describing the evolution of the controlled one-dimensional harmonic oscillator,

$$
i \frac{\partial \psi}{\partial t}(t, x)=-\frac{\partial^{2} \psi}{\partial x^{2}}(t, x)+\left(x^{2}-u(t) W(x)\right) \psi(t, x),
$$

where $\psi$ is the wave function depending on the time $t$ and on a space variable $x \in \mathbf{R}=\Omega$. Recall that $u(\cdot)$ is a piecewise-continuous function with values in a subset $U$ of $\mathbf{R}$. Notice that the potential corresponding to the uncontrolled Schrödinger operator is $V(x)=x^{2}$. The control system (6.2) has been studied, among others, by Mirrahimi and Rouchon who proved its non-controllability in the case where $W$ is the identity function (see [28]).

As a consequence of Theorem 3.2, the spectrum of $-\Delta+V$ is discrete. Its explicit expression is

$$
\left\{\lambda_{k}=2 k+1 \mid k \geqslant 0\right\}
$$

and therefore $\lambda_{k+1}-\lambda_{k}$ are $\mathbf{Q}$-linearly dependent. Each $\lambda_{k}$ is a simple eigenvalue whose corresponding eigenfunction is

$$
\phi_{k}(x)=\frac{1}{\sqrt{k ! 2^{k} \sqrt{\pi}}} e^{-\frac{x^{2}}{2}} H_{k}(x)
$$

where $H_{k}(x)=(-1)^{k} e^{x^{2}} \frac{d^{k}}{d x^{k}} e^{-x^{2}}$ is the $k$ th Hermite polynomial.

In order to apply Corollary 6.3 we would like first of all to ensure that the elements

$$
b_{k k}=\frac{(-1)^{k}}{k ! 2^{k} \sqrt{\pi}} \int_{\mathbf{R}} W(x) H_{k}(x) \frac{d^{k}}{d x^{k}} e^{-x^{2}} d x, \quad k \geqslant 0,
$$

are Q-linearly independent. Notice that for $W(x)=x$ (i.e., the non-controllable case pointed out by Mirrahimi and Rouchon), since each function $\phi_{k}^{2}$ is even, $b_{k k}=\int W \phi_{k}^{2}=0$.

The existence of controlled potentials $W$ for which the elements of $\left(b_{k k}\right)_{k \in \mathbf{N}}$ are $\mathbf{Q}$-linearly independent can be easily inferred from the linear independence of the functions $\phi_{k}^{2}$. The proposition below provides some explicit $W$ with such a property (and such that the corresponding Schrödinger equation is controllable). The potentials $W$ will be chosen in $L^{\infty}(\mathbf{R})$ and therefore, as already remarked in Section 3, the corresponding solutions in the sense (2.2) coincide with mild or strong solutions, depending on the regularity of the initial condition.

\section{Proposition 6.4.}

(1) If $W$ is even, then system (6.2) is not approximately controllable.

(2) If $W$ has the form $W: x \mapsto e^{a x^{2}+b x+c}$, with $a, b, c \in \mathbf{R}$ such that $a<0$ and the two numbers $\sqrt{1-a}$ and $b$ are algebraically independent, then system (6.2) is approximately controllable, provided that $U$ has non-empty interior.

Proof. Since each function $\phi_{k}$ has the same parity as the integer $k$, then $\phi_{k} \phi_{j}$ has the same parity as the integer $j+k$. If $W$ is even, then (6.1) shows that for every $(j, k)$ such that $j+k$ is odd, $b_{j k}=0$. Applying Remark 4.2, one sees that the spaces spanned by the sets $\left\{\phi_{k} \mid k\right.$ even $\}$ and $\left\{\phi_{k} \mid k\right.$ odd $\}$ are invariant by the dynamics of system (6.2). In particular, there is no way to steer system (6.2) from $\phi_{1}$ to a point $\varepsilon$-close to $\phi_{2}$ if $\varepsilon$ is smaller than $\sqrt{2}$. This proves (1).

In order to prove (2) let us apply Corollary 6.3 (with $U$ playing the role of $\tilde{U}$ and $\mathbf{R}$ the role of $U$ ). Let $W$ have the special form $W: x \mapsto e^{a x^{2}+b x+c}$. Up to a multiplication of $W$ by the strictly positive real number $e^{\frac{b^{2}}{4(a-1)}-c}$, we may assume without loss of generality that

$$
c=\frac{b^{2}}{4(a-1)} \text {. }
$$

Using the specific expression (6.3) of $\phi_{k}$ in the definition of $b_{j k}$ we can write

$$
b_{j k}=(-1)^{j} \sigma_{j} \sigma_{k} \int_{\mathbf{R}} e^{a x^{2}+b x+c} H_{k}(x) \frac{d^{j}}{d x^{j}} e^{-x^{2}} d x,
$$


with $\sigma_{l}=1 / \sqrt{l ! 2^{l} \sqrt{\pi}}, l=k, j$. Notice that $H_{k}$ is a polynomial with rational coefficients and of degree $k$, whose leading coefficient is equal to $2^{k}$. Integrating by parts $j$ times, we get

$$
b_{j k}=\sigma_{j} \sigma_{k} \int_{\mathbf{R}} e^{(a-1) x^{2}+b x+c} P_{j, k}(x) d x
$$

where $P_{j, k}$ is a polynomial of degree $j+k$. Define $\left(g_{m}^{j, k}\right)_{m=0}^{j+k}$ through

$$
P_{j, k}(x)=\sum_{m=0}^{j+k} g_{m}^{j, k} x^{m} .
$$

Each $g_{m}^{j, k}$ can be seen as the evaluation at $b$ of a polynomial $G_{m}^{j, k}$ with coefficients in $\mathbf{Q}[a]$ whose degree is less than or equal to $j$. If $m \in\{k, k+1, \ldots, k+j\}$ then $G_{m}^{j, k}$ has exactly degree $j+k-m$ and the coefficient corresponding to the monomial of order $j+k-m$ is $2^{m} a^{m-k}$.

The renormalization of $c$ performed above is such that $(a-1) x^{2}+b x+c=(a-1)\left(x+\frac{b}{2 \sqrt{1-a}}\right)^{2}$. Hence, the change of variables $y=\sqrt{1-a}\left(x+\frac{b}{2 \sqrt{1-a}}\right)$ yields

$$
b_{j k}=\frac{\sigma_{j} \sigma_{k}}{\sqrt{1-a}} \int_{\mathbf{R}} e^{-y^{2}} P_{j, k}\left(\frac{y}{\sqrt{1-a}}-\frac{b}{2(a-1)}\right) d y .
$$

Due to the remarks made above on the coefficients of $P_{j, k}$, we have

$$
\begin{aligned}
P_{j, k}\left(\frac{y}{\sqrt{1-a}}-\frac{b}{2(a-1)}\right) & =\sum_{m=k}^{j+k} 2^{m} a^{m-k} b^{j+k-m}\left(\frac{-b}{2(a-1)}\right)^{m}+Q_{j, k}(b, y) \\
& =\frac{(-1)^{k}}{(a-1)^{k}} \frac{1-\left(\frac{a}{a-1}\right)^{j+1}}{1-\frac{a}{a-1}} b^{j+k}+Q_{j, k}(b, y)
\end{aligned}
$$

where $Q_{j, k}$ is a polynomial with coefficients in $\mathbf{Q}(\sqrt{1-a})$ ( $\supset \mathbf{Q}[a]$ ) and of degree smaller than $j+k$ in its first variable. Notice that the coefficient multiplying $b^{j+k}$ in (6.6) is different from zero.

For every $m \geqslant 0$ the integral $\int_{\mathbf{R}} e^{-y^{2}} y^{m} d y$ is equal to zero if $m$ is odd and to $\Gamma\left(\frac{m+1}{2}\right)=\frac{m !}{2^{m}\left(\frac{m}{2}\right) !} \sqrt{\pi}$ if $m$ is even, where $\Gamma$ is the Euler gamma function.

Therefore, if $j+k$ is even,

$$
b_{j k}=\frac{\sigma_{j} \sigma_{k} \sqrt{\pi}}{\sqrt{1-a}} S_{j, k}(b)
$$

where $S_{j, k}$ is a polynomial with coefficients in $\mathbf{Q}(\sqrt{1-a})$ of degree exactly $j+k$.

Since $b$ is transcendental over $\mathbf{Q}(\sqrt{1-a})$ then $b_{j k} \neq 0$ as soon as $j$ and $k$ have the same parity. Moreover, the elements of the sequence $\left(\Lambda_{k}^{\prime}(0)\right)_{k \geqslant 0}=\left(b_{k k}\right)_{k} \geqslant 0$ are $\mathbf{Q}$-linearly independent.

To conclude the proof let us check that each matrix $\left(b_{j k}\right)_{j, k=0}^{n}$ is connected. Fix $j, k \in\{0, \ldots, n\}$. We should prove the existence of a sequence $r_{1}, \ldots, r_{l} \in\{0, \ldots, n\}$ such that $b_{j r_{1}} b_{r_{1} r_{2}} \cdots b_{r_{l-1} r_{l}} b_{r_{l} k} \neq 0$. If $j$ and $k$ have the same parity then we are done since $b_{j k} \neq 0$. Otherwise, a simple computation and the normalization (6.5) show that

$$
b_{01}=\frac{b}{\sqrt{2}(1-a)^{3 / 2}} \neq 0
$$

and we can conclude by taking $\left\{r_{1}, r_{2}\right\}=\{0,1\}$.

\section{3. $3 D$ potential well}

Consider the Schrödinger equation

$$
i \frac{\partial \psi}{\partial t}(t, x)=-\Delta \psi(t, x)+u(t) W(x) \psi(t, x),
$$


where the wave function $\psi$ depends on the time $t$ and on three space variables $x_{1}, x_{2}, x_{3}$ with $\left(x_{1}, x_{2}, x_{3}\right) \in\left(0, l_{1}\right) \times$ $\left(0, l_{2}\right) \times\left(0, l_{3}\right)=\Omega$ and satisfies the Dirichlet boundary condition $\left.\psi\right|_{\partial \Omega}=0$. Notice that the potential corresponding to the uncontrolled Schrödinger operator is $V(x)=0$. For every $W$ measurable bounded, solutions in the sense (2.2) coincide with mild or strong solutions, depending on the regularity of the initial condition.

The spectrum of the Schrödinger operator is

$$
\left\{\lambda_{k_{1}, k_{2}, k_{3}}=\pi^{2}\left(\frac{k_{1}^{2}}{l_{1}^{2}}+\frac{k_{2}^{2}}{l_{2}^{2}}+\frac{k_{3}^{2}}{l_{3}^{2}}\right) \mid k_{1}, k_{2}, k_{3} \geqslant 1\right\} .
$$

For the sake of simplicity, assume that $\left(l_{1} l_{2}\right)^{2},\left(l_{1} l_{3}\right)^{2}$, and $\left(l_{2} l_{3}\right)^{2}$ are $\mathbf{Q}$-linearly independent, so that all the eigenvalues are simple and the perturbation result appearing in Theorem 6.1 can be applied. (The case of multiple eigenvalues can be treated similarly, applying a refined perturbation argument as the one used in [6].)

The normalized eigenfunction corresponding to $\lambda_{k_{1}, k_{2}, k_{3}}$ is given, up to sign, by

$$
\phi_{k_{1}, k_{2}, k_{3}}\left(x_{1}, x_{2}, x_{3}\right)=\frac{2^{\frac{3}{2}}}{\sqrt{l_{1} l_{2} l_{3}}} \sin \left(\frac{k_{1} x_{1} \pi}{l_{1}}\right) \sin \left(\frac{k_{2} x_{2} \pi}{l_{2}}\right) \sin \left(\frac{k_{3} x_{3} \pi}{l_{3}}\right) .
$$

Proposition 6.5. Let $\left(l_{1} l_{2}\right)^{2},\left(l_{1} l_{3}\right)^{2}$, and $\left(l_{2} l_{3}\right)^{2}$ be Q-linearly independent and define $W\left(x_{1}, x_{2}, x_{3}\right)=e^{\alpha_{1} x_{1}+\alpha_{2} x_{2}+\alpha_{3} x_{3}}$ with $\alpha_{1}, \alpha_{2}, \alpha_{3} \in \mathbf{R}$. Assume that $\alpha_{1}, \alpha_{2}, \alpha_{3}$ are non-zero and that $\left(\pi / \alpha_{1} l_{1}\right)^{2},\left(\pi / \alpha_{2} l_{2}\right)^{2},\left(\pi / \alpha_{3} l_{3}\right)^{2}$ are algebraically independent. Then the control system (6.7) is approximately controllable.

Before starting the proof of Proposition 6.5 let us show the following technical result.

Lemma 6.6. Let $\beta$ be a real number transcendental over a field $\mathbf{F}$ with $\mathbf{Q} \subset \mathbf{F} \subset \mathbf{R}$. Then the elements of the family $\left(\frac{1}{1+q \beta}\right)_{q \in \mathbf{Q}}$ are $\mathbf{F}$-linearly independent.

Proof. Fix $N \in \mathbf{N}$ and $N$ distinct numbers $q_{1}, \ldots, q_{N} \in \mathbf{Q} \backslash\{0\}$. Assume that for some $f_{1}, \ldots, f_{N}$ in $\mathbf{F}$

$$
\sum_{k=1}^{N} f_{k} \frac{1}{1+q_{k} \beta}=0
$$

We have to prove that $f_{1}=f_{2}=\cdots=f_{N}=0$. Multiplying $(6.8)$ by $\prod_{k=1}^{N}\left(1+q_{k} \beta\right)$ we get

$$
\sum_{k=1}^{N} f_{k}\left(\sum_{r=0}^{N-1} s_{k, r} \beta^{r}\right)=0
$$

where $s_{k, 0}=1$ and, for $r \geqslant 1$,

$$
s_{k, r}=\sum_{\substack{1 \leqslant j_{1}<j_{2}<\cdots<j_{r} \leqslant N \\ j_{1}, \ldots, j_{r} \neq k}} q_{j_{1}} q_{j_{2}} \cdots q_{j_{r}}
$$

By hypothesis, all coefficients of the left-hand side of (6.9), seen as a polynomial in $\beta$, are equal to zero. Hence, $\left(f_{1}, \ldots, f_{N}\right) S_{N}=(0, \ldots, 0)$ where

$$
S_{N}=\left(\begin{array}{ccc}
s_{1,0} & \cdots & s_{1, N-1} \\
\vdots & & \vdots \\
s_{N, 0} & \cdots & s_{N, N-1}
\end{array}\right)
$$

A computation shows that $\operatorname{det}\left(S_{N}\right)=\Pi_{1 \leqslant j<k \leqslant N}\left(q_{k}-q_{j}\right)$. Hence, $S_{N}$ is invertible and therefore $f_{1}=f_{2}=\cdots=$ $f_{N}=0$.

Proof of Proposition 6.5. Theorem 6.1 and Fubini's theorem imply that the eigenvalues $\Lambda_{k_{1}, k_{2}, k_{3}}(\mu)$ of $-\Delta+\mu W$ on $\Omega$ for the Dirichlet boundary value problem satisfy 


$$
\begin{aligned}
\Lambda_{k_{1}, k_{2}, k_{3}}^{\prime}(0) & =\frac{64\left(e^{\alpha_{1} l_{1}}-1\right)\left(e^{\alpha_{2} l_{2}}-1\right)\left(e^{\alpha_{3} l_{3}}-1\right) k_{1}{ }^{2} k_{2}{ }^{2} k_{3}{ }^{2} \pi^{6}}{\alpha_{1} l_{1} \alpha_{2} l_{2} \alpha_{3} l_{3}\left(4 \pi^{2} k_{1}^{2}+\alpha_{1}^{2} l_{1}^{2}\right)\left(4 \pi^{2} k_{2}^{2}+\alpha_{2}^{2} l_{2}^{2}\right)\left(4 \pi^{2} k_{3}^{2}+\alpha_{3}^{2} l_{3}^{2}\right)} \\
& =C k_{1}^{2} k_{2}^{2} k_{3}^{2} \frac{1}{\left(\frac{4 \pi^{2}}{\alpha_{1}^{2} l_{1}^{2}} k_{1}^{2}+1\right)\left(\frac{4 \pi^{2}}{\alpha_{2}^{2} l_{2}^{2}} k_{2}^{2}+1\right)\left(\frac{4 \pi^{2}}{\alpha_{3}^{2} l_{3}^{2}} k_{3}^{2}+1\right)},
\end{aligned}
$$

where

$$
C=\frac{64\left(e^{\alpha_{1} l_{1}}-1\right)\left(e^{\alpha_{2} l_{2}}-1\right)\left(e^{\alpha_{3} l_{3}}-1\right) \pi^{6}}{\left(\alpha_{1} l_{1} \alpha_{2} l_{2} \alpha_{3} l_{3}\right)^{3}} .
$$

Let $\beta_{j}=4 \pi^{2} /\left(\alpha_{j}^{2} l_{j}^{2}\right), j=1,2,3$. The $\mathbf{Q}$-linear independence of the elements of $\left(\Lambda_{k_{1}, k_{2}, k_{3}}^{\prime}(0)\right)_{k_{1}, k_{2}, k_{3} \in \mathbf{N}}$ is obtained from the expression above thanks to three nested applications of Lemma 6.6 with $\mathbf{F}=\mathbf{Q}\left(\beta_{1}, \beta_{2}\right)$ and $\beta=\beta_{3}, \mathbf{F}=$ $\mathbf{Q}\left(\beta_{1}\right)$ and $\beta=\beta_{2}$, and $\mathbf{F}=\mathbf{Q}$ and $\beta=\beta_{1}$. In order to complete the proof, let us check that every matrix $B^{(n)}$ is connected. (The conclusion then follows from Corollary 6.3.) A straightforward computation shows that for every triples of positive integers $\left(k_{1}, k_{2}, k_{3}\right)$ and $\left(h_{1}, h_{2}, h_{3}\right)$ the integral

$$
\int_{\Omega} e^{\alpha_{1} x_{1}+\alpha_{2} x_{2}+\alpha_{3} x_{3}} \phi_{k_{1}, k_{2}, k_{3}}\left(x_{1}, x_{2}, x_{3}\right) \phi_{h_{1}, h_{2}, h_{3}}\left(x_{1}, x_{2}, x_{3}\right) d x_{1} d x_{2} d x_{3}
$$

is different from zero, i.e., every element of $B^{(n)}$ is non-zero.

\section{Acknowledgements}

The authors are grateful to Andrei Agrachev for inspiring this work and to George Weiss, Marius Tucsnak, Riccardo Adami, Anne de Roton, Takéo Takahashi for helpful discussions.

\section{References}

[1] R. Adami, U. Boscain, Controllability of the Schrödinger equation via intersection of eigenvalues, in: Proceedings of the 44th IEEE Conference on Decision and Control, December 12-15, 2005, pp. 1080-1085.

[2] A. Agrachev, T. Chambrion, An estimation of the controllability time for single-input systems on compact Lie groups, ESAIM Control Optim. Calc. Var. 12 (3) (2006) 409-441.

[3] A. Agrachev, S. Kuksin, A. Sarychev, A. Shirikyan, On finite-dimensional projections of distributions for solutions of randomly forced 2D Navier-Stokes equations, Ann. Inst. H. Poincaré Probab. Statist. 43 (4) (2007) 399-415.

[4] A.A. Agrachev, Y.L. Sachkov, Control Theory from the Geometric Viewpoint, Encyclopaedia of Mathematical Sciences, vol. 87, SpringerVerlag, Berlin, 2004, Control Theory and Optimization, II.

[5] A.A. Agrachev, A.V. Sarychev, Controllability of 2D Euler and Navier-Stokes equations by degenerate forcing, Commun. Math. Phys. 265 (3) (2006) 673-697.

[6] J.H. Albert, Genericity of simple eigenvalues for elliptic PDE’s, Proc. Amer. Math. Soc. 48 (1975) 413-418.

[7] F. Albertini, D. D'Alessandro, Notions of controllability for bilinear multilevel quantum systems, IEEE Trans. Automat. Control 48 (8) (2003) 1399-1403.

[8] C. Altafini, Controllability of quantum mechanical systems by root space decomposition of $s u(N)$, J. Math. Phys. 43 (5) (2002) $2051-2062$.

[9] C. Altafini, Controllability properties for finite dimensional quantum Markovian master equations, J. Math. Phys. 44 (6) (2003) $2357-2372$.

[10] J.M. Ball, J.E. Marsden, M. Slemrod, Controllability for distributed bilinear systems, SIAM J. Control Optim. 20 (4) (1982) $575-597$.

[11] L. Baudouin, O. Kavian, J.-P. Puel, Regularity for a Schrödinger equation with singular potentials and application to bilinear optimal control, J. Differential Equations 216 (1) (2005) 188-222.

[12] K. Beauchard, Local controllability of a 1-D Schrödinger equation, J. Math. Pures Appl. (9) 84 (7) (2005) 851-956.

[13] K. Beauchard, J.-M. Coron, Controllability of a quantum particle in a moving potential well, J. Funct. Anal. 232 (2) (2006) $328-389$.

[14] A. Borzì, E. Decker, Analysis of a leap-frog pseudospectral scheme for the Schrödinger equation, J. Comput. Appl. Math. 193 (1) (2006) 65-88.

[15] U. Boscain, T. Chambrion, G. Charlot, Nonisotropic 3-level quantum systems: complete solutions for minimum time and minimum energy, Discrete Contin. Dyn. Syst. Ser. B 5 (4) (2005) 957-990 (electronic).

[16] U. Boscain, G. Charlot, Resonance of minimizers for $n$-level quantum systems with an arbitrary cost, ESAIM Control Optim. Calc. Var. 10 (4) (2004) 593-614 (electronic).

[17] U. Boscain, P. Mason, Time minimal trajectories for a spin 1/2 particle in a magnetic field, J. Math. Phys. $47(6)(2006) 29,062101$.

[18] J.-M. Coron, Control and Nonlinearity, Mathematical Surveys and Monographs, vol. 136, American Mathematical Society, Providence, RI, 2007. 
[19] D. D'Alessandro, Introduction to Quantum Control and Dynamics, Applied Mathematics and Nonlinear Science Series, Chapman, Hall/CRC, Boca Raton, FL, 2008.

[20] E.B. Davies, Spectral Theory and Differential Operators, Cambridge Studies in Advanced Mathematics, vol. 42, Cambridge University Press, Cambridge, 1995.

[21] A. Henrot, Extremum Problems for Eigenvalues of Elliptic Operators, Frontiers in Mathematics, Birkhäuser Verlag, Basel, 2006.

[22] P. Hübler, J. Bargon, S.J. Glaser, Nuclear magnetic resonance quantum computing exploiting the pure spin state of para hydrogen, J. Chem. Phys. 113 (6) (2000) 2056-2059.

[23] K. Ito, K. Kunisch, Optimal bilinear control of an abstract Schrödinger equation, SIAM J. Control Optim. 46 (1) (2007) $274-287$ (electronic).

[24] V. Jurdjevic, H.J. Sussmann, Control systems on Lie groups, J. Differential Equations 12 (1972) 313-329.

[25] T. Kato, Perturbation Theory for Linear Operators, Die Grundlehren der mathematischen Wissenschaften, Band 132, Springer-Verlag, New York, 1966.

[26] N. Khaneja, S.J. Glaser, R. Brockett, Sub-Riemannian geometry and time optimal control of three spin systems: quantum gates and coherence transfer, Phys. Rev. A 65 (3) (2002) 11, 032301.

[27] M. Mirrahimi, Lyapunov control of a particle in a finite quantum potential well, in: Proceedings of the 45th IEEE Conference on Decision and Control, December 13-15, 2006.

[28] M. Mirrahimi, P. Rouchon, Controllability of quantum harmonic oscillators, IEEE Trans. Automat. Control 49 (5) (2004) $745-747$.

[29] A. Peirce, M. Dahleh, H. Rabitz, Optimal control of quantum mechanical systems: Existence, numerical approximations, and applications, Phys. Rev. A 37 (1988) 4950-4964.

[30] V. Pierfelice, Strichartz estimates for the Schrödinger and heat equations perturbed with singular and time dependent potentials, Asymptotic Anal. 47 (1-2) (2006) 1-18.

[31] H. Rabitz, H. de Vivie-Riedle, R. Motzkus, K. Kompa, Wither the future of controlling quantum phenomena? Science 288 (2000) $824-828$.

[32] M. Reed, B. Simon, Methods of Modern Mathematical Physics. IV. Analysis of Operators, Academic Press (Harcourt Brace Jovanovich Publishers), New York, 1978.

[33] F. Rellich, Perturbation Theory of Eigenvalue Problems, Assisted by J. Berkowitz. With a preface by Jacob T. Schwartz. Gordon Breach Science Publishers, New York, 1969.

[34] I. Rodnianski, W. Schlag, Time decay for solutions of Schrödinger equations with rough and time-dependent potentials, Invent. Math. 155 (3) (2004) 451-513.

[35] S.S. Rodrigues, Navier-Stokes equation on the rectangle controllability by means of low mode forcing, J. Dynam. Control Syst. 12 (4) (2006) 517-562.

[36] P. Rouchon, Control of a quantum particle in a moving potential well, in: Lagrangian and Hamiltonian Methods for Nonlinear Control 2003, IFAC, Laxenburg, 2003, pp. 287-290.

[37] Y.L. Sachkov, Controllability of invariant systems on Lie groups and homogeneous spaces, Dynamical systems, 8, J. Math. Sci. (New York) 100 (4) (2000) 2355-2427.

[38] M. Shapiro, P. Brumer, Principles of the Quantum Control of Molecular Processes, Wiley-VCH, 2003, pp. 250.

[39] G. Tenenbaum, M. Tucsnak, K. Ramdani, T. Takahashi, A spectral approach for the exact observability of infinite dimensional systems with skew-adjoint generator, J. Funct. Anal., 2007.

[40] G. Turinici, On the controllability of bilinear quantum systems, in: M. Defranceschi, C. Le Bris (Eds.), Mathematical Models and Methods for ab initio Quantum Chemistry, in: Lecture Notes in Chemistry, vol. 74, Springer, 2000.

[41] E. Zuazua, Remarks on the controllability of the Schrödinger equation, in: Quantum Control: Mathematical and Numerical Challenges, in: CRM Proc. Lecture Notes, vol. 33, Amer. Math. Soc., Providence, RI, 2003, pp. 193-211. 